\title{
Droplet Solutions in the Diblock Copolymer Problem with Skewed Monomer Composition
}

\author{
Xiaofeng Ren * \\ Department of Mathematics and Statistics \\ Utah State University \\ Logan, UT 84322-3900, USA
}

\author{
Juncheng Wei ${ }^{\dagger}$ \\ Department of Mathematics \\ Chinese University of Hong Kong \\ Hong Kong, PRC
}

June 5, 2005

\begin{abstract}
A droplet solution characterizes the lamellar phase of a diblock copolymer when the two composing monomers maintain a skewed ratio. We study the threshold case where the free energy of a droplet solution is comparable to the free energy of the constant solution. Using a Lyapunov-Schmidt reduction approach, adapted to calculus of variations, we prove the existence of a free energy local minimizer with a given number of droplets. Also determined are the free energy, the droplet location, and the droplet size.
\end{abstract}

\section{Introduction}

A diblock copolymer molecule is a linear sub-chain of $N_{A} A$-monomers grafted covalently to another sub-chain of $N_{B} B$-monomers. Because of the repulsion between the unlike monomers, the different type sub-chains tend to segregate, but as they are chemically bonded in chain molecules, segregation of sub-chains cannot lead to a macroscopic phase separation. Only a local micro-phase separation occurs: micro-domains rich in $A$ and $B$ emerge. These micro-domains form morphology patterns/phases in a larger scale.

The Ohta-Kawasaki [15] free energy of an incompressible diblock copolymer melt is a functional of the $A$-monomer density field. Let $u(x)$ be the relative $A$-monomer number density at point $x$ in the sample. When there is high $A$-monomer concentration at $x, u(x)$ is close to 1 ; when there is high concentration of $B$-monomers at $x, u(x)$ is close to 0 . A value of $u(x)$ between 0 and 1 means that a mixture of $A$ - and $B$-monomers occupies $x$. In one-dimension the re-scaled, dimensionless free energy of the system is

$$
I(u)=\int_{0}^{1}\left[\frac{\epsilon^{2}}{2}\left|u^{\prime}\right|^{2}+W(u)+\frac{\sigma}{2}\left|(-\Delta)^{-1 / 2}(u-a)\right|^{2}\right] d x .
$$

${ }^{*}$ Corresponding author. Phone: 1435 797-0755, Fax 1435 797-1822, E-mail: ren@math.usu.edu

${ }^{\dagger}$ Supported in part by a Direct Grant from CUHK and an earmarked Grant of RGC of Hong Kong. 
The functional $I$ is defined in the admissible set

$$
\mathcal{A}=\left\{u \in W^{1,2}(0,1): \bar{u}=a\right\}
$$

where $\bar{u}=\int_{0}^{1} u d x$ is the average of $u$.

The term $W(u)$ is the internal energy field. We take it to be a smooth double well potential of equal depth. It has global minimum value 0 achieved at 0 and 1 . We assume for simplicity that $W$ is smooth, grows at least quadratically at $\pm \infty$, and symmetric about $1 / 2: W(u)=W(1-u) .0$ and 1 are non-degenerate: $W^{\prime \prime}(0)=W^{\prime \prime}(1)>0$. An example of $W$ is $W(u)=\frac{1}{4}\left(u^{2}-u\right)^{2}$.

The other two terms in (1.1) give the entropy of the system. The peculiar nonlocal term is due to the fact that molecules in a diblock copolymer are connected long chains. It models a type of nonlocal interaction known as the Coulomb interaction. Mathematically we view $(-\Delta)^{-1}$ as a bounded positive operator from $\left\{\zeta \in L^{2}(0,1): \bar{\zeta}=0\right\}$ to $\left\{\xi \in W^{2,2}(0,1): \bar{\xi}=0, \xi^{\prime}(0)=\xi^{\prime}(1)=0\right\}$ : $\xi=(-\Delta)^{-1} \zeta$ if

$$
-\xi^{\prime \prime}=\zeta \text { in }(0,1), \xi^{\prime}(0)=\xi^{\prime}(1)=0, \quad \bar{\xi}=0 .
$$

Then $(-\Delta)^{-1 / 2}$ is the positive square root of $(-\Delta)^{-1}$.

Three positive parameters $\epsilon, \sigma$ and $a$ appear in (1.1). $\epsilon$ is always a small parameter, and $\sigma$ is proportional to the size of the sample. In earlier works $a$ has been taken to be a fixed constant, independent of $\epsilon$, in $(0,1)$. Then one may choose $\sigma$ to be of order $\epsilon$ and apply the $\Gamma$-limit theory (De Giorgi [4], Modica and Mortola [11], Modica [10], and Kohn and Sternberg [9]). This was the approach used in Ren and Wei [16]. In terms of the number of $A$-monomers and $B$-monomers, $N_{A}$ and $N_{B}$, in each chain molecule

$$
a=\frac{N_{A}}{N_{A}+N_{B}} .
$$

$N_{A}$ and $N_{B}$ are both large integers. The condition that $a \in(0,1)$ is independent of $\epsilon$ means that $N_{A}$ and $N_{B}$ are comparable which we denote by

$$
N_{A} \sim N_{B} .
$$

In [16] we found local minimizers that characterize the lamellar phase of a diblock copolymer. A typical local minimizer is shown in Figure 1. It is close to 0 in some intervals and close to 1 in other intervals. The two types of intervals are comparable in length, and they are separated by sharp interfaces.

In this paper we consider a case where $N_{A}$ and $N_{B}$ are large but not comparable. Namely we assume a skewed relationship that $N_{A}$ and $\sqrt{N_{B}}$ are comparable:

$$
N_{A} \sim \sqrt{N_{B}} .
$$

Hence $N_{A}$ is a lot smaller than $N_{B}$. The condition (1.6) turns out to be equivalent to (cf. Choksi and Ren [3])

$$
a \sim \epsilon^{1 / 2}, \sigma \sim 1 .
$$

Therefore throughout this paper we take the positive $a$ to be

$$
a=\epsilon^{1 / 2} a_{0} \text {, where } a_{0}>0 \text { is independent of } \epsilon,
$$

and assume that $\sigma$ is independent of $\epsilon$. 


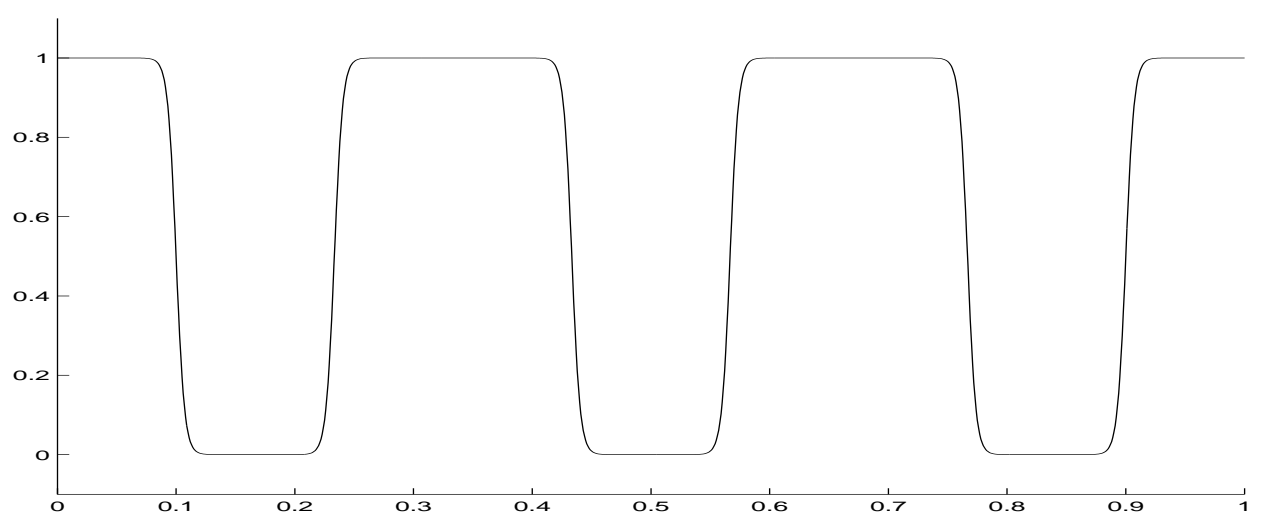

Figure 1: A local minimizer with 6 interfaces in the case (1.5).

In addition to its physical relevance, the condition (1.7) generates some interesting mathematical questions. Define a nonlinear operator

$$
S(u)=-\epsilon^{2} u^{\prime \prime}+f(u)-\overline{f(u)}+\sigma(-\Delta)^{-1}(u-a)
$$

where $f=W^{\prime}$. If $W$ is the particular $W(u)=\frac{1}{4}\left(u^{2}-u\right)^{2}$, then $f$ is $f(u)=u\left(u-\frac{1}{2}\right)(u-1)$. $S$ is defined from the space

$$
\mathcal{X}=\left\{u \in W^{2,2}(0,0): \bar{u}=a, u^{\prime}(0)=u^{\prime}(1)=0,\right\}
$$

to the space

$$
\mathcal{Y}=\left\{z \in L^{2}(0,1): \bar{z}=0\right\}
$$

The Euler-Lagrange equation of $I$ is

$$
S(u)=0, u \in \mathcal{X}
$$

The standard $\Gamma$-limit approach used in [16] to solve (1.12) is to identify a limiting functional of $\epsilon^{-1} I$. The $\Gamma$-limit theory asserts that if one can find an isolated local minimizer of the limiting functional, then for small $\epsilon, I$ also has a local minimizer nearby. To identify the limiting functional in [16], the value $a$ must be $\epsilon$ independent. The parameter range (1.8) can not be dealt with by straightforward $\Gamma$-limit argument. Instead we will use a Lyapunov-Schmidt reduction approach, adapted specifically for calculus of variations. The latter method is more complex because one must understand the linearization of $S$ at some carefully constructed approximate solutions. One may roughly regard the $\Gamma$-limit theory as a $C^{0}$ singular limit theory and the Lyapunov-Schmidt theory as a $C^{2}$ theory.

The solutions of (1.12) that we are interested in will model the lamellar phase of a diblock copolymer. They must be non-constant local minimizers of $I$. Because of the skewed value (1.8), we want a solution to be close to 0 everywhere except in some small intervals where the solution is close to 1 (Figure 2). We call these small intervals droplets, and call the solution a droplet solution. The condition (1.7) ensures that (1.6) is met, and the sample is of the proper size so a finite number of droplets are observed. We will show that the size of the droplets is of order $\epsilon^{1 / 2}$.

The parameter range (1.7) may also be an important threshold. The energy of a droplet solution will turn out to be of order $\epsilon$. It is of the same order as the energy of the constant solution $u=a$. 


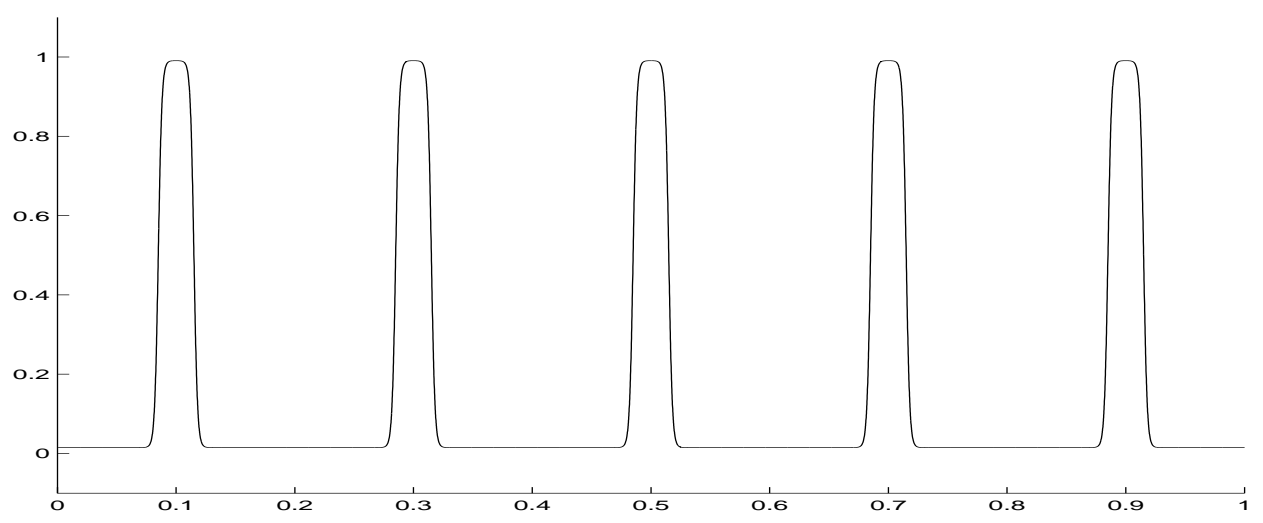

Figure 2: A local minimizer with 5 droplets in the case (1.6).

If we consider a parameter range where $a \ll \epsilon^{1 / 2}$, then the energy of the constant solution will be of lower order. Then it is doubtful that the Ohta-Kawasaki model will allow any non-constant morphology.

Finally we wish to gain some crucial ideas before we study the 2- and 3- dimensional versions of the skewed monomer composition cases. There one finds the more interesting cylindrical and spherical morphology phases (cf. Bates and Fredrickson [1]).

The main result of this paper is the following theorem.

Theorem 1.1 For each positive integer $N$ there exists a local minimizer of $I$ with $N$ small droplets when $\epsilon$ is sufficiently small. The free energy of the local minimizer is

$$
\epsilon\left[2 N \tau+f^{\prime}(0) a_{0}^{2}\left(\frac{1}{2}-\frac{\operatorname{coth} \beta-\operatorname{csch} \beta}{\beta}\right)\right]+o(\epsilon)
$$

where $\tau=\int_{0}^{1} \sqrt{2 W(z)} d z$ and $\beta=\frac{\sqrt{\sigma}}{N \sqrt{f^{\prime}(0)}}$.

More information about the $N$-droplet local minimizer will be available as we prove the theorem. In particular we will know the location and the size of the droplets.

In our Lyapunov-Schmidt reduction approach to prove Theorem 1.1, we construct a manifold $\mathcal{M}$ of approximate solutions $w_{\xi}$ parameterized by a $2 N$-vector $\xi$, together with a fiber $w_{\xi}+\mathcal{F}_{\xi}$ at each $w_{\xi}$ (Section 2). The manifold $\mathcal{M}$ and its fibers $w_{\xi}+\mathcal{F}_{\xi}$ form a local decomposition of $\mathcal{X}$. In Section 3 we find $\phi_{\xi}$ so that $w_{\xi}+\phi_{\xi}$ solves (1.12) in the fiber direction. In Section 4 we prove that $w_{\xi}+\phi_{\xi}$ locally minimizes $I$ in a similar fiber $w_{\xi}+\mathcal{P}_{\xi}$. To our knowledge this is the first time in the literature of Lyapunov-Schmidt reduction when the fiber solution is connected to the variational structure. Finally in Section 5 we find a particular $\xi$ so that at this $\xi, w_{\xi}+\phi_{\xi}$ solves (1.12) in $\mathcal{X}$ and it is a local minimizer of $I$. A few remarks are included in Section 6 and some technical proofs are in the appendix.

The mathematical literature on the diblock copolymer problem includes Nishiura and Ohnishi [13], Ohnishi et al [14], Ren and Wei [18, 17, 21, 19, 24, 22, 25], Choksi [2], Fife and Hilhorst [5], 
Henry [7], and Teramoto and Nishiura [26]. More information on the model (1.1) and its extension to triblock copolymers may be found in Nakazawa and Ohta [12], and Ren and Wei [20].

In the notations of this paper, a quantity's dependence on $\epsilon$ is not emphasized. For example we write $I$ instead of $I_{\epsilon}$ and $S$ instead of $S_{\epsilon}$. However whenever a quantity is independent of $\epsilon$, we point out the fact. The $L^{\infty}$ norm will simply be denoted by $\|\cdot\|$, and the $L^{2}(0,1)$ norm denoted by $\|\cdot\|_{2}$. The $L^{2}(0,1)$ inner product is denoted by $\langle\cdot, \cdot\rangle$. All projection operations used in the paper are derived from this inner product. Constants like $C, C_{1}, C_{2}, \ldots$, are all independent of $\epsilon$. They may vary from place to place.

\section{The approximate manifold and its fibers}

Let $\xi_{1}, \xi_{2}, \ldots, \xi_{2 N}$ be $2 N$ numbers in $(0,1)$, satisfying $\xi_{1}<\xi_{2}<\ldots<\xi_{2 N}$. Given $\xi=\left(\xi_{1}, \xi_{2}, \ldots, \xi_{2 N}\right)$ we will build an approximate solution whose interfaces are at the $\xi_{j}$ 's. An interface is a point where the approximate solution changes between 0 and 1 . Mathematically we require that the approximate solution is equal to $1 / 2$ at an interface $\xi_{j}$. The centers of the droplets are at $\zeta_{k}$ :

$$
\zeta_{k}=\frac{\xi_{2 k-1}+\xi_{2 k}}{2}, k=1,2, \ldots, N
$$

and the half widths, scaled by $\epsilon^{1 / 2}$, of the droplets are $l_{k}$ :

$$
l_{k}=\frac{\xi_{2 k}-\xi_{2 k-1}}{2 \epsilon^{1 / 2}}, k=1,2, \ldots, N
$$

Hence the first droplet is bounded by the interfaces $\xi_{1}$ and $\xi_{2}$, and the second droplet is bounded by the interfaces $\xi_{3}$ and $\xi_{4}$, etc. We can express $\xi$ in terms of $\zeta=\left(\zeta_{1}, \zeta_{2}, \ldots, \zeta_{N}\right)$ and $l=\left(l_{1}, l_{2}, \ldots, l_{N}\right)$ :

$$
\xi_{2 k}=\zeta_{k}+\epsilon^{1 / 2} l_{k}, \quad \xi_{2 k-1}=\zeta_{k}-\epsilon^{1 / 2} l_{k}, k=1,2, \ldots, N .
$$

The exact set $\Lambda \subset \mathbf{R}^{2 N}$ in which $\xi$ varies is

$$
\Lambda=\left\{\xi \in \mathbf{R}^{2 N}: \zeta_{1}>\delta, \zeta_{2}-\zeta_{1}>\delta, \ldots, \zeta_{N}-\zeta_{N-1}>\delta, 1-\zeta_{N}>\delta, \frac{1}{\delta}>l_{k}>\delta, k=1, \ldots, N\right\}
$$

where $\xi$ is related to $(\zeta, l)$ via $(2.3)$. In $(2.4) \delta$ is a positive constant independent of $\epsilon$. It is small enough so that a particular $\left(\zeta^{0}, l^{0}\right)$ defined in Lemma 5.2, Section 5 , satisfies the inequalities in (2.4). Because $l_{k}$ is of order 1 , the size of each droplet is of order $\epsilon^{1 / 2}$.

We construct a manifold of approximate solutions parameterized by $\xi \in \Lambda$. First define

$$
s_{\xi}(x)=0 \text { in }\left(0, \xi_{1}\right), 1 \text { in }\left(\xi_{1}, \xi_{2}\right), 0 \text { in }\left(\xi_{2}, \xi_{3}\right), \ldots, 1 \text { in }\left(\xi_{2 N-1}, \xi_{2 N}\right), 0 \text { in }\left(\xi_{2 N}, 1\right)
$$

which gives a profile away from the interfaces. The interface profile is the solution $H$ of the differential equation

$$
-H^{\prime \prime}+f(H)=0, H(-\infty)=0, H(\infty)=1, H(0)=\frac{1}{2} .
$$

$H(t)$ approaches 0 (or 1 respectively) exponentially fast as $t$ tends to $-\infty$ (or $\infty$ respectively) in the sense that there exist positive $C_{1}, C_{2}$ so that

$$
0<H(t)<C_{1} e^{C_{2} t} \text { if } t<0 \text {, and } 0<1-H(t)<C_{1} e^{-C_{2} t} \text { if } t>0 .
$$


Near $\xi_{j}$ we use $H\left(\left(x-\xi_{j}\right) / \epsilon\right)$ if $j$ is odd, or $H\left(-\left(x-\xi_{j}\right) / \epsilon\right)$ if $j$ is even. Then $s_{\xi}$ and $H$ must be connected by a smooth cut-off function $\chi$ to make

$$
w_{\xi, 1}(x)=\sum_{j=1}^{2 N} \chi\left(x-\xi_{j}\right) H\left((-1)^{j+1} \frac{x-\xi_{j}}{\epsilon}\right)+\left(1-\sum_{j=1}^{2 N} \chi\left(x-\xi_{j}\right)\right) s_{\xi}(x) .
$$

where $\chi$ is defined to be

$$
\chi(x)=\left\{\begin{array}{lll}
1 & \text { in } & \left(-\epsilon^{\alpha}, \epsilon^{\alpha}\right) \\
0 & \text { in } & \mathbf{R} \backslash\left(-2 \epsilon^{\alpha}, 2 \epsilon^{\alpha}\right)
\end{array} .\right.
$$

The exponent $\alpha$ in (2.9) satisfies

$$
\frac{1}{2}<\alpha<1
$$

$\chi$ satisfies

$$
\chi=O(1), \chi^{\prime}=O\left(\epsilon^{-\alpha}\right), \chi^{\prime \prime}=O\left(\epsilon^{-2 \alpha}\right) .
$$

It turns out that $w_{\xi, 1}$ is not accurate enough to be an approximate solution. A correction to $w_{\xi, 1}$ is needed. Let $w_{\xi, 2}$ be the solution of

$$
f^{\prime}(0) w_{\xi, 2}+\sigma(-\Delta)^{-1}\left(w_{\xi, 1}+w_{\xi, 2}-a\right)=f^{\prime}(0) \overline{w_{\xi, 2}}, \overline{w_{\xi, 1}+w_{\xi, 2}-a}=0
$$

which may be written as

$$
-D w_{\xi, 2}^{\prime \prime}+w_{\xi, 2}+w_{\xi, 1}-a=0, w_{\xi, 2}^{\prime}(0)=w_{\xi, 2}^{\prime}(1)=0 .
$$

Here

$$
D=\frac{f^{\prime}(0)}{\sigma} .
$$

$w_{\xi, 2}$ is easily estimated. We denote the Green function of the last differential equation by $G_{D}$, i.e.

$$
-D \frac{G^{2}(x, y)}{\partial x^{2}}+G(x, y)=\delta(x-y), \quad \frac{\partial G(0, y)}{\partial x}=\frac{\partial G(1, y)}{\partial x}=0
$$

where $\delta(\cdot)$ is the delta measure.

\section{Lemma 2.1}

$$
w_{\xi, 2}(x)=\epsilon^{1 / 2}\left(a_{0}-\sum_{j=1}^{N} 2 l_{j} G_{D}\left(x, \zeta_{j}\right)\right)+O(\epsilon) .
$$

In particular $w_{\xi, 2}=O\left(\epsilon^{1 / 2}\right)$.

Proof. Note that $\epsilon^{-1 / 2} w_{\xi, 1}$ approaches $\sum_{j=1}^{K} 2 l_{j} \delta\left(\cdot-\zeta_{j}\right)$ in the sense of distributions, as $\epsilon \rightarrow 0$. The lemma then follows easily.

Now we set an approximate solution

$$
w_{\xi}=w_{\xi, 1}+w_{\xi, 2} \in \mathcal{X} .
$$

As $\xi$ varies in $\Lambda w_{\xi}$ forms a $2 N$-dimensional manifold which we denote by $\mathcal{M}$ embedded in $\mathcal{X}$ :

$$
\mathcal{M}=\left\{w_{\xi} \in \mathcal{X}: \xi \in \Lambda\right\} .
$$

The next lemma shows how accurate $w_{\xi}$ is. 
Lemma 2.2 $S\left(w_{\xi}\right)=\left(f^{\prime}\left(w_{\xi, 1}\right)-f^{\prime}(0)\right) w_{\xi, 2}+O(\epsilon)$.

Proof. First note that

$$
\begin{aligned}
\overline{f\left(w_{\xi}\right)} & =\int_{0}^{1} f\left(w_{\xi, 1}\right) d x+\int_{0}^{1} f^{\prime}\left(w_{\xi, 1}\right) w_{\xi, 2} d x+O(\epsilon) \\
& =\int_{0}^{1} f^{\prime}\left(w_{\xi, 1}\right) w_{\xi, 2} d x+O(\epsilon) \\
& =f^{\prime}(0) \overline{w_{\xi, 2}}+\int_{0}^{1}\left[f^{\prime}\left(w_{\xi, 1}\right)-f^{\prime}(0)\right] w_{\xi, 2} d x+O(\epsilon) \\
& =f^{\prime}(0) \overline{w_{\xi, 2}}+O(\epsilon) .
\end{aligned}
$$

To reach (2.22) we have used the fact that $f^{\prime}\left(w_{\xi, 1}\right)-f^{\prime}(0)=O(1)$ only on $\epsilon$-size neighborhoods of $\xi_{j}$. Elsewhere $w_{\xi, 1}$ is exponentially close to 0 or 1 , and hence $f^{\prime}\left(w_{\xi, 1}\right)-f^{\prime}(0)$ is exponentially close to 0 . Then using $(2.12)$ we find that

$$
\begin{aligned}
S\left(w_{\xi}\right) & =-\epsilon^{2} w_{\xi}^{\prime \prime}+f\left(w_{\xi}\right)+\sigma(-\Delta)^{-1}\left(w_{\xi}-a\right)-f^{\prime}(0) \overline{w_{\xi, 2}}+O(\epsilon) \\
& =-\epsilon^{2} w_{\xi}^{\prime \prime}+f\left(w_{\xi}\right)-f^{\prime}(0) w_{\xi, 2}+O(\epsilon) \\
& =-\epsilon^{2} w_{\xi, 1}^{\prime \prime}-\epsilon^{2} w_{\xi, 2}^{\prime \prime}+f\left(w_{\xi, 1}\right)+f^{\prime}\left(w_{\xi, 1}\right) w_{\xi, 2}-f^{\prime}(0) w_{\xi, 2}+O(\epsilon) \\
& =\left(-\epsilon^{2} w_{\xi, 1}^{\prime \prime}+f\left(w_{\xi, 1}\right)\right)+\left(f^{\prime}\left(w_{\xi, 1}\right)-f^{\prime}(0)\right) w_{\xi, 2}+O(\epsilon) \\
& =\left(f^{\prime}\left(w_{\xi, 1}\right)-f^{\prime}(0)\right) w_{\xi, 2}+O(\epsilon) .
\end{aligned}
$$

This proves the lemma.

Corollary 2.3 $S\left(w_{\xi}\right)=O\left(\epsilon^{1 / 2}\right)$.

Proof. It follows from Lemmas 2.1 and 2.2 .

Note that Lemma 2.2 is a stronger statement than Corollary 2.3. Lemma 2.2 implies that $S\left(w_{\xi}\right)$ is $O\left(\epsilon^{1 / 2}\right)$ in $\epsilon$ size neighborhoods of $\xi_{j}$. Elsewhere $S\left(w_{\xi}\right)$ is $O(\epsilon)$.

For each $j=1,2, \ldots, 2 N$, let us define

$$
h_{j}(x)=H^{\prime}\left(\frac{x-x_{j}}{\epsilon}\right) \kappa\left(\frac{x-x_{j}}{\sqrt{\epsilon}}\right)-\overline{H^{\prime}\left(\frac{x-x_{j}}{\epsilon}\right) \kappa\left(\frac{x-x_{j}}{\sqrt{\epsilon}}\right)}=H^{\prime}\left(\frac{x-x_{j}}{\epsilon}\right)-\epsilon+O\left(e^{-C / \epsilon}\right) .
$$

where $\kappa$ is a smooth, even cut-off function

$$
\kappa(s)= \begin{cases}1 & \text { if }|s| \leq 1 \\ 0 & \text { if }|s| \geq 2\end{cases}
$$

Here $O\left(e^{-C / \epsilon}\right)$ is an exponentially small quantity with respect to $\epsilon$ because of the exponentially fast decay rate of $H^{\prime}: H^{\prime}(t) \leq C_{1} e^{-C_{2}|t|}$. Therefore $h_{j}^{\prime}(0)=h_{j}^{\prime}(1)=0,\left\|h_{j}^{\prime}-\epsilon^{-1} H^{\prime \prime}\left(\frac{{ }^{-}-x_{j}}{\epsilon}\right)\right\|_{\infty}=$ $O\left(\epsilon^{-C / \epsilon}\right)$, and $\left\|h_{j}^{\prime \prime}-\epsilon^{-2} H^{\prime \prime \prime}\left(\frac{\cdot x_{j}}{\epsilon}\right)\right\|_{\infty}=O\left(\epsilon^{-C / \epsilon}\right)$. Not emphasized in the notation, the $h_{j}$ 's defined in (2.28) depend on $\xi$.

At each $w_{\xi}$ of the manifold we define the space

$$
\mathcal{F}_{\xi}=\left\{\phi \in W^{2,2}(0,1): \bar{\phi}=0, \phi^{\prime}(0)=\phi^{\prime}(1)=0, \quad \phi \perp h_{j}, j=1,2, \ldots, 2 N\right\}
$$


where $\perp$ is defined from the $L^{2}(0,1)$ inner product. Then $w_{\xi}+\mathcal{F}_{\xi}$ is a subset of $\mathcal{X}$, which we call the $\xi$-fiber of $\mathcal{M}$ in $\mathcal{X}$. Define $\mathcal{E}_{\xi}$ to be the subspace

$$
\mathcal{E}_{\xi}=\left\{q \in L^{2}(0,1): \bar{q}=0, q \perp h_{j}, j=1,2, \ldots, 2 N\right\}
$$

of $\mathcal{Y}$. Let the projection to $\mathcal{E}_{\xi}$ be $\pi_{\xi}$

$$
\pi_{\xi}: \mathcal{Y} \rightarrow \mathcal{E}_{\xi}
$$

\section{Solvability in fibers}

At each $w_{\xi}$ we look for a $\phi_{\xi} \in \mathcal{F}_{\xi}$ so that

$$
\pi_{\xi} \circ S\left(w_{\xi}+\phi_{\xi}\right)=0
$$

This means that we solve $S(u)=0$ in the fiber direction. For each $\phi \in \mathcal{F}_{\xi}$ we expand

$$
S\left(w_{\xi}+\phi\right)=S\left(w_{\xi}\right)+L_{\xi}(\phi)+R_{\xi}(\phi)
$$

where

$$
L_{\xi}(\phi)=-\epsilon^{2} \phi^{\prime \prime}+f^{\prime}\left(w_{\xi}\right) \phi-\overline{f^{\prime}\left(w_{\xi}\right) \phi}+\sigma(-\Delta)^{-1} \phi
$$

is the linearization of $S$ at $w_{\xi}$, and

$$
R_{\xi}(\phi)=f\left(w_{\xi}+\phi\right)-f\left(w_{\xi}\right)-f^{\prime}\left(w_{\xi}\right) \phi-\overline{f\left(w_{\xi}+\phi\right)-f\left(w_{\xi}\right)-f^{\prime}\left(w_{\xi}\right) \phi} .
$$

Then (3.1) is written as

$$
\pi_{\xi} \circ S\left(w_{\xi}\right)+\pi_{\xi} \circ L_{\xi}\left(\phi_{\xi}\right)+\pi_{\xi} \circ R_{\xi}\left(\phi_{\xi}\right)=0
$$

Regarding the the linear operator $\pi_{\xi} \circ L_{\xi}$ :

$$
\pi_{\xi} \circ L_{\xi}: \mathcal{F}_{\xi} \rightarrow \mathcal{E}_{\xi}
$$

we have the following lemma.

Lemma 3.1 1. There exists $C_{1}>0$ independent of $\epsilon$ such that $\|\phi\| \leq C_{1}\left\|\pi_{\xi} \circ L_{\xi}(\phi)\right\|$ for all $\phi \in \mathcal{F}_{\xi}$. In particular $\pi_{\xi} \circ L_{\xi}$ is one-to-one from $\mathcal{F}_{\xi}$ to $\mathcal{E}_{\xi}$.

2. $\pi_{\xi} \circ L_{\xi}$ is onto from $\mathcal{F}_{\xi}$ to $\mathcal{E}_{\xi}$.

Proof. To prove part 1 we argument by contradiction. Suppose the conclusion is false. Then there exists $\psi \in \mathcal{F}_{\xi}$ for each $\epsilon$ such that $\|\psi\|=1$ and along a subsequence of $\epsilon \rightarrow 0$,

$$
\left\|\pi_{\xi} \circ L_{\xi}(\psi)\right\| \rightarrow 0
$$

Write (3.7) as

$$
-\epsilon^{2} \psi^{\prime \prime}+f^{\prime}\left(w_{\xi}\right) \psi-\overline{f^{\prime}\left(w_{\xi}\right) \psi}+\sigma(-\Delta)^{-1} \psi-\sum_{j=1}^{2 N} \beta_{j} h_{j}=o(1)
$$


for some $\beta_{j} \in \mathbf{R}$. We multiply the last equation by $\psi$ and integrate. Denoting $\varphi=(-\Delta)^{-1} \psi$, we deduce

$$
\int_{0}^{1}\left[\epsilon^{2}\left|\psi^{\prime}\right|^{2}+f^{\prime}\left(w_{\xi}\right) \psi^{2}+\sigma\left|\varphi^{\prime}\right|^{2}\right] d x=o(1)
$$

Since $f^{\prime}\left(w_{\xi}\right)$ may only be negative in $\epsilon$-size neighborhoods of $\xi_{j}$, the last equation implies

$$
\int_{0}^{1}\left|\varphi^{\prime}\right|^{2} d x=o(1)
$$

The Sobolev embedding theory yields

$$
\|\varphi\|=o(1)
$$

We must estimate the size of $\beta_{j}$. To this end we multiply (3.8) by $h_{k}$ and integrate. Then

$$
\int_{0}^{1}\left[\left(-\epsilon^{2} \psi^{\prime \prime}+f^{\prime}\left(w_{\xi}\right) \psi+\sigma \varphi\right) h_{k}\right] d x+\sum_{j=1}^{2 N} \beta_{j}\left\langle h_{j}, h_{k}\right\rangle=o(\epsilon) .
$$

Simple calculations simplify the second part on the left side, so

$$
\int_{0}^{1}\left(-\epsilon^{2} \psi^{\prime \prime}+f^{\prime}\left(w_{\xi}\right)+\sigma \varphi\right) h_{k} d x+\sum_{j=1}^{2 N} \beta_{j}\left(\epsilon \tau \delta_{j k}+O\left(\epsilon^{2}\right)\right)=o(\epsilon)
$$

where $\delta_{j k}=1$ if $j \neq k$ and 0 if $j=k$. Also we have used the fact that

$$
\tau=\int_{\mathbf{R}}\left(H^{\prime}\right)^{2} d t
$$

The first part of the left side of (3.13) is estimated as follows:

$$
\begin{aligned}
\int_{0}^{1} & {\left[\left(-\epsilon^{2} \psi^{\prime \prime}+f^{\prime}\left(w_{\xi}\right) \psi+\sigma \varphi\right) h_{k}\right] d x } \\
& =\int_{0}^{1}\left(-\epsilon^{2} h_{k}^{\prime \prime} \psi+f^{\prime}\left(w_{\xi}\right) \psi h_{k}\right) d x+\int_{0}^{1} \sigma \varphi h_{k} d x \\
& =\int_{0}^{1}\left[\left(-H^{\prime \prime \prime}\left(\frac{x-\xi_{k}}{\epsilon}\right)+f^{\prime}\left(w_{\xi}\right) H\left(\frac{x-\xi_{k}}{\epsilon}\right)\right) \psi-f^{\prime}\left(w_{\xi}\right) \psi \epsilon\right] d x+\int_{0}^{1} \sigma \varphi h_{k} d x \\
& =O(\epsilon) .
\end{aligned}
$$

This simplifies (3.13) to

$$
\sum_{j=1}^{2 N} \beta_{j}\left(\epsilon \tau \delta_{j k}+O\left(\epsilon^{2}\right)\right)=O(\epsilon)
$$

Hence

$$
\beta_{j}=O(1)
$$


Let $y \in[0,1]$ such that, without the loss of generality, $\psi(y)=\|\psi\|=1$. We claim that $y-\xi_{j}=$ $O(\epsilon)$ for some $j$. Otherwise, at $y$, because of (3.11),

$$
\begin{aligned}
L_{\xi}(\psi)(y) & =-\epsilon^{2} \psi^{\prime \prime}(y)+f^{\prime}\left(w_{\xi}(y)\right) \psi(y)-\overline{f^{\prime}\left(w_{\xi}\right) \psi}+\sigma\left((-\Delta)^{-1} \psi\right)(y) \\
& \geq 0+f^{\prime}\left(w_{\xi}(y)\right)-\overline{\left(f^{\prime}\left(w_{\xi}\right)-f^{\prime}(0)\right) \psi}+\sigma\left((-\Delta)^{-1} \psi\right)(y) \\
& =f^{\prime}\left(w_{\xi}(y)\right)+\sigma \varphi(y)+O(\epsilon)=f^{\prime}\left(w_{\xi}(y)\right)+o(1) \\
& =f^{\prime}(0)+o(1) .
\end{aligned}
$$

Combining (3.20) and (3.24), we obtain

$$
\pi_{\xi} \circ L_{\xi}(\psi)(y) \geq f^{\prime}(0)-\sum_{j=1}^{2 N} \beta_{j} h_{j}(y)+o(1) \geq f^{\prime}(0)+o(1)
$$

which contradicts (3.7).

We have thus proved that $y-\xi_{j}=O(\epsilon)$ for some $j$, along a subsequence of $\epsilon \rightarrow 0$. Define $\Psi(t)=\psi\left(\xi_{j}+\epsilon t\right)$. Then (3.8), (3.11), and (3.20) imply

$$
-\Psi^{\prime \prime}+f^{\prime}\left(w_{\xi}\left(\xi_{j}+\epsilon t\right)\right) \Psi-\beta_{j} H^{\prime} \rightarrow 0
$$

uniformly on any compact subset of $\mathbf{R}$. From here we may pass the limit and find $\Psi_{\infty}$ and $\beta_{j}^{0}$ so that $\Psi \rightarrow \Psi_{\infty}$ in $C_{l o c}^{2}(\mathbf{R})$ and $\beta_{j} \rightarrow \beta_{j}^{0}$. Moreover $\Psi_{\infty} \neq 0$ since $\Psi\left(\left(y-\xi_{j}\right) / \epsilon\right)=1$, and

$$
-\Psi_{\infty}^{\prime \prime}+f^{\prime}(H) \Psi_{\infty}-\beta_{j}^{0} H^{\prime}=0 .
$$

If we multiply the last equation by $H^{\prime}$ and integrate over $\mathbf{R}$, we find $\beta_{j}^{0}=0$. Then $\Psi_{\infty}$ satisfies

$$
-\Psi_{\infty}^{\prime \prime}+f^{\prime}(H) \Psi_{\infty}=0 .
$$

The bounded solutions of this equation are scalar multiples of $H^{\prime}$. Hence $\Psi_{\infty}=c H^{\prime}$ for some $c \neq 0$.

On the other hand, sine $\psi \in \mathcal{F}_{\xi}$ means that $\psi \perp h_{j}$ and $\bar{\psi}=0$, we deduce that

$$
0=\left\langle\psi, h_{j}\right\rangle=\epsilon \int_{-\xi_{j} / \epsilon}^{\left(1-\xi_{j}\right) / \epsilon} \Psi(t)\left(H^{\prime}(t)-O\left(e^{-C / \epsilon}\right)\right) d t=\epsilon\left(c \int_{\mathbf{R}}\left(H^{\prime}(t)\right)^{2} d t+o(1)\right)
$$

which is impossible. We have thus proved part 1 of the lemma.

To prove part 2 of the lemma we need to solve

$$
\pi_{\xi} \circ L_{\xi}(\phi)=p
$$

in $\mathcal{F}_{\xi}$ for any given $p \in \mathcal{E}_{\xi}$. By applying $\pi_{\xi} \circ(-\Delta)^{-1}$ to both sides of (3.30) we consider the equation

$$
\pi_{\xi} \circ(-\Delta)^{-1} \circ \pi_{\xi} \circ L_{\xi}(\phi)=\pi_{\xi} \circ(-\Delta)^{-1} p .
$$

The linear operator $\pi_{\xi} \circ(-\Delta)^{-1} \circ \pi_{\xi} \circ L_{\xi}$ on the left side maps from $\mathcal{F}_{\xi}$ to itself. For this operator $F_{\xi}$ is viewed as a Banach space whose norm is inherited from the $W^{2,2}(0,1)$ norm. The operator has the form

$$
\epsilon^{2} \text { (Identity Operator) + Compact Operator. }
$$


According to the Fredholm Alternative, (3.31) is solvable if

$$
\pi_{\xi} \circ(-\Delta)^{-1} \circ \pi_{\xi} \circ L_{\xi}(\phi)=0
$$

only has the trivial solution. To see this we write (3.33) as

$$
(-\Delta)^{-1} \circ \pi_{\xi} \circ L_{\xi}(\phi)=\sum_{j=1}^{2 N} \alpha_{j} h_{j}
$$

for some $\alpha_{j} \in \mathbf{R}$. Apply $-\Delta$ to the last equation to find

$$
\pi_{\xi} \circ L_{\xi}(\phi)=\sum_{j=1}^{2 N} \alpha_{j}\left(-h_{j}^{\prime \prime}\right) .
$$

We multiply it by $h_{k}$ and integrate to deduce

$$
0=\sum_{j=1}^{2 N} \alpha_{j} \int_{0}^{1} h_{j}^{\prime} h_{k}^{\prime} d x=\alpha_{k} \int_{0}^{1}\left|h_{k}^{\prime}\right|^{2} d x, k=1,2, \ldots, 2 N,
$$

which implies that $\alpha_{j}=0, j=1,2, \ldots, 2 N$. Then (3.35) becomes

$$
\pi_{\xi} \circ L_{\xi}(\phi)=0 .
$$

The first part of the lemma implies that $\phi=0$.

Hence (3.31) is solvable, i.e. for any $p \in \mathcal{E}_{\xi}$ there exist $\phi \in \mathcal{F}_{\xi}$ and $\beta_{j} \in \mathbf{R}$ such that

$$
(-\Delta)^{-1} \circ \pi_{\xi} \circ L_{\xi}(\phi)=(-\Delta)^{-1} p+\sum_{j=1}^{2 N} \beta_{j} h_{j} .
$$

Apply $-\Delta$ to the last equation to deduce

$$
\pi_{\xi} \circ L_{\xi}(\phi)=p+\sum_{j=1}^{2 N} \beta_{j}\left(-h_{j}^{\prime \prime}\right) .
$$

We multiply by $h_{k}$ and integrate to obtain

$$
0=\sum_{j=1}^{2 N} \beta_{j} \int_{0}^{1} h_{j}^{\prime} h_{k}^{\prime} d x=\beta_{k} \int_{0}^{1}\left|h_{k}^{\prime}\right|^{2} d x, k=1,2, \ldots, 2 N,
$$

which implies that $\beta_{j}=0$, for all $j=1,2, \ldots, 2 N$. Then (3.39) becomes (3.30).

We are now ready to solve (3.1).

Lemma 3.2 There exists $\phi_{\xi} \in \mathcal{F}_{\xi}$ with $\left\|\phi_{\xi}\right\|=O\left(\epsilon^{1 / 2}\right)$ so that $\pi_{\xi} \circ S\left(w_{\xi}+\phi_{\xi}\right)=0$. 
Proof. We write (3.5) in a fixed point form:

$$
\phi_{\xi}=\left(\pi_{\xi} \circ L_{\xi}\right)^{-1}\left(-\pi_{\xi} \circ S\left(w_{\xi}\right)-\pi_{\xi} \circ R_{\xi}\left(\phi_{\xi}\right)\right)
$$

We define the operator $T_{\xi}$ from $\mathcal{D}\left(T_{\xi}\right)$ to itself

$$
T_{\xi}(\phi)=\left(\pi_{\xi} \circ L_{\xi}\right)^{-1}\left(-\pi_{\xi} \circ S\left(w_{\xi}\right)-\pi_{\xi} \circ R_{\xi}(\phi)\right)
$$

where the domain $\mathcal{D}\left(T_{\xi}\right)$ of $T_{\xi}$ is

$$
\mathcal{D}\left(T_{\xi}\right)=\left\{\phi \in L^{\infty}(0,1): \bar{\phi}=0, \phi \perp h_{j}, j=1,2, \ldots, 2 N\right\}
$$

Let $\mathcal{B}_{\xi}$ be a closed ball in $\mathcal{D}\left(T_{\xi}\right)$ defined by

$$
\mathcal{B}_{\xi}=\left\{\phi \in \mathcal{D}\left(T_{\xi}\right):\|\phi\| \leq C_{2} \epsilon^{1 / 2}\right\}
$$

where $C_{2}$ is a constant independent of $\epsilon$ to be determined soon. For every $\phi \in \mathcal{B}_{\xi}$, by Corollary 2.3

$$
\begin{aligned}
\left\|T_{\xi}(\phi)\right\| & \leq C_{1}\left\|\pi_{\xi} \circ S\left(w_{\xi}\right)\right\|+C_{1}\left\|\pi_{\xi} \circ R_{\xi}(\phi)\right\| \\
& \leq C_{3} \epsilon^{1 / 2}+C_{5}(1+O(\|\phi\|))\|\phi\|^{2} \\
& \leq C_{3} \epsilon^{1 / 2}+C_{6} C_{2}^{2}\left(1+C_{2} \epsilon^{1 / 2}\right) \epsilon
\end{aligned}
$$

where we have estimated $R_{\xi}(\phi)$ as follows:

$$
\left\|R_{\xi}(\phi)\right\| \leq 2\left\|f\left(w_{\xi}+\phi\right)-f\left(w_{\xi}\right)-f^{\prime}\left(w_{\xi}\right) \phi\right\| \leq C_{4}(1+O(\|\phi\|))\|\phi\|^{2}
$$

for some $C_{4}$ depending on $f$ only. In (3.47) the constants $C_{3}$ and $C_{6}$ are again independent of $\epsilon$. If we choose $C_{2}$ to be sufficiently large, then when $\epsilon$ is small enough (3.47) is bounded by $C_{2} \epsilon^{1 / 2}$. Therefore by choosing such $C_{2}$ we see that $\mathcal{D}\left(T_{\xi}\right)$ maps $\mathcal{B}_{\xi}$ to itself.

Next we prove that $T_{\xi}$ is a contraction mapping in $\mathcal{D}\left(T_{\xi}\right)$. Take $\phi_{1}$ and $\phi_{2}$ in $\mathcal{D}\left(T_{\xi}\right)$. Then

$$
\begin{aligned}
\left\|T_{\xi}\left(\phi_{1}\right)-T_{\xi}\left(\phi_{2}\right)\right\| & \leq C_{1}\left\|\pi_{\xi} \circ\left(R_{\xi}\left(\phi_{1}\right)-R_{\xi}\left(\phi_{2}\right)\right)\right\| \leq C_{7}\left\|R_{\xi}\left(\phi_{1}\right)-R_{\xi}\left(\phi_{2}\right)\right\| \\
& \leq C_{8}\left\|f\left(w_{\xi}+\phi_{1}\right)-f\left(w_{\xi}+\phi_{2}\right)-f^{\prime}\left(w_{\xi}\right)\left(\phi_{1}-\phi_{2}\right)\right\| \\
& \leq C_{8}\left\|f^{\prime}\left(w_{\xi}+\phi_{2}+\theta\left(\phi_{1}-\phi_{2}\right)\right)\left(\phi_{1}-\phi_{2}\right)-f^{\prime}\left(w_{\xi}\right)\left(\phi_{1}-\phi_{2}\right)\right\| \\
& \leq C_{8}\left\|f^{\prime}\left(w_{\xi}+\phi_{2}+\theta\left(\phi_{1}-\phi_{2}\right)\right)-f^{\prime}\left(w_{\xi}\right)\right\|\left\|\phi_{1}-\phi_{2}\right\| \\
& \leq O\left(\left\|\phi_{1}\right\|+\left\|\phi_{2}\right\|\right)\left\|\phi_{1}-\phi_{2}\right\| \\
& \leq C_{9} \epsilon^{1 / 2}\left\|\phi_{1}-\phi_{2}\right\|
\end{aligned}
$$

which implies that $T_{\xi}$ is a contraction mapping if $\epsilon$ is sufficiently small. In these estimates $\theta=$ $\theta(x) \in(0,1)$ comes from the mean value theorem.

\section{Stability in fibers}

In this section we connect the fiber solution $w_{\xi}+\phi_{\xi}$ to the variational structure $I$. The manifold $\mathcal{M}$ is also embedded in the admissible set $\mathcal{A}$ defined in (1.2). At each $w_{\xi}$ we let

$$
\mathcal{P}_{\xi}=\left\{\psi \in W^{1,2}(0,1): \bar{\psi}=0, \psi \perp h_{j}, j=1,2, \ldots, 2 N\right\} .
$$


Then $w_{\xi}+\mathcal{P}_{\xi}$ is a subset of $\mathcal{A}$, which we call the $\xi$-fiber of $\mathcal{M}$ in $\mathcal{A}$. Note that $\mathcal{F}_{\xi} \subset \mathcal{P}_{\xi}$.

In this section we show that $w_{\xi}+\phi_{\xi}$ found in the last section locally minimizes $I$ in $w_{\xi}+\mathcal{P}_{\xi}$. We first study the linearization of $S$ at $w_{\xi}+\phi_{\xi}$. Set

$$
\lambda=\inf \left\{\int_{0}^{1}\left[\epsilon^{2}\left(\psi^{\prime}\right)^{2}+f^{\prime}\left(w_{\xi}+\phi_{\xi}\right) \psi^{2}+\sigma\left|(-\Delta)^{-1 / 2} \psi\right|^{2}\right] d x: \psi \in \mathcal{P}_{\xi}, \int_{0}^{1} \psi^{2} d x=1\right\} .
$$

Lemma $4.1 \liminf _{\epsilon \rightarrow 0} \lambda>0$.

Proof. In this proof we denote $w_{\xi}+\phi_{\xi}$ by $g_{\xi}$. We argue by contradiction. Assume $\liminf _{\epsilon \rightarrow 0} \lambda \leq 0$. Clearly $\lambda$ is achieved by a $\psi$ in $\mathcal{P}_{\xi}$. Scaling $\psi$ by a constant multiple, we may assume that $\|\psi\|=1$ and $\psi$ satisfies

$$
-\epsilon^{2} \psi^{\prime \prime}+f^{\prime}\left(g_{\xi}\right) \psi-\overline{f^{\prime}\left(g_{\xi}\right) \psi}+\sigma(-\Delta)^{-1} \psi=\lambda \psi+\sum_{j=1}^{2 N} \beta_{j} h_{j}, \psi^{\prime}(0)=\psi^{\prime}(1)=0,
$$

for some $\beta_{j} \in \mathbf{R}$. Note that $\bar{\psi}=0, \psi \perp h_{j}$, and $\|\psi\|=1$, but $\int_{0}^{1} \psi^{2} d x$ is no longer equal to 1 .

First multiply $(4.3)$ by $\psi$, denote $\varphi=(-\Delta)^{-1} \psi$, and integrate to obtain

$$
\int_{0}^{1}\left[\epsilon^{2}\left(\psi^{\prime}\right)^{2}+f^{\prime}\left(g_{\xi}\right) \psi^{2}+\sigma\left(\varphi^{\prime}\right)^{2}\right] d x=\lambda \int_{0}^{1} \psi^{2} d x
$$

Since $f^{\prime}\left(g_{\xi}\right)$ may only be negative in $\epsilon$-size neighborhoods of $\xi_{j}$, we deduce from (4.4) that

$$
O(\epsilon)+\sigma \int_{0}^{1}\left(\varphi^{\prime}\right)^{2} d x \leq \lambda_{+}
$$

where

$$
\lambda_{+}=\left\{\begin{array}{lll}
0 & \text { if } & \lambda \leq 0 \\
\lambda & \text { if } & \lambda>0
\end{array} .\right.
$$

The assumption $\liminf _{\epsilon \rightarrow 0} \lambda \leq 0$ implies that

$$
\lambda_{+}=o(1)
$$

By the Sobolev embedding theory, (4.5) and (4.7) imply that

$$
\|\varphi\|=O\left(\sqrt{\lambda_{+}+\epsilon}\right)=o(1)
$$

Next multiply (4.3) by $h_{k}$ and integrate to deduce

$$
\int_{0}^{1}\left[-\epsilon^{2} \psi^{\prime \prime} h_{k}+f^{\prime}\left(g_{\xi}\right) \psi h_{k}+\sigma \varphi h_{k}\right] d x=\sum_{j=1}^{2 N} \beta_{j} \epsilon\left(\tau \delta_{j k}+O(\epsilon)\right) .
$$

As in the argument leading to (3.20) we find that

$$
\beta_{j}=O(1), j=1,2, \ldots, 2 N .
$$


Thirdly we let $y \in[0,1]$ so that $\psi(y)=\|\psi\|=1$. We show that $y$ must be in an $\epsilon$-size neighborhood of some $\xi_{k}$. Otherwise (4.3) is not valid at $y$, for as in (3.24)

$$
f^{\prime}(0)+o(1) \leq-\epsilon^{2} \psi^{\prime \prime}(y)+f^{\prime}\left(g_{\xi}(y)\right) \psi(y)-\overline{f^{\prime}\left(g_{\xi}\right) \psi}+\sigma \varphi(y)=\lambda \psi(y)+\sum_{j=1}^{2 N} \beta_{j} h_{j}(y)=\lambda+o(1)
$$

with the help of (4.8) and (4.10). But this is impossible since we have assumed that $\liminf \lambda \leq 0$.

Finally we assume that $y-\xi_{k}=O(\epsilon)$ for some $k$ and look for a contradiction. $\stackrel{\epsilon \rightarrow 0}{\text { Let }} \Psi(t)=$ $\psi\left(\xi_{j}+\epsilon t\right)$. Then

$$
-\Psi^{\prime \prime}(t)+f^{\prime}\left(g_{\xi}\left(\xi_{j}+\epsilon t\right)\right) \Psi+o(1)=\lambda \Psi(t)+\sum_{j=1}^{2 N} \beta_{j} h_{j}\left(\xi_{k}+\epsilon t\right), t \in\left(-\frac{\xi_{k}}{\epsilon}, \frac{1-\xi_{k}}{\epsilon}\right) .
$$

As in the proof of Lemma $3.1 \Psi$ converges to $\Psi_{\infty}$ in $C_{l o c}^{2}(\mathbf{R}) . \Psi_{\infty} \neq 0$ since $\Psi\left(\left(y-\xi_{k}\right) / \epsilon\right)=1$, and $\Psi_{\infty}$ satisfies

$$
-\Psi_{\infty}^{\prime \prime}+f^{\prime}(H) \Psi_{\infty}=\left(\liminf _{\epsilon \rightarrow 0} \lambda\right) \Psi_{\infty}+\beta_{k}^{0} H^{\prime}
$$

where $\beta_{k}^{0}=\lim \beta_{k}$. Since $\psi \perp h_{k}$ and $\bar{\psi}=0$,

$$
0=\int_{0}^{1} \psi h_{k} d x=\int_{0}^{1} \psi\left(H^{\prime}\left(\frac{x-\xi_{\epsilon}}{\epsilon}\right)+O\left(e^{-C / \epsilon}\right)\right) d x=\epsilon \int_{\mathbf{R}} \Psi_{\infty}(t) H^{\prime}(t) d t+o(\epsilon) .
$$

Therefore

$$
\int_{\mathbf{R}} \Psi_{\infty} H^{\prime} d t=0
$$

We multiply (4.13) by $H^{\prime}$ and integrate. Because of (4.15) we obtain from (4.13) that

$$
0=\beta_{k}^{0} \int_{\mathbf{R}}\left(H^{\prime}\right)^{2} d t \text {, i.e. } \beta_{k}^{0}=0 .
$$

Then (4.13) becomes

$$
-\Psi_{\infty}^{\prime \prime}+f^{\prime}(H) \Psi_{\infty}=\left(\liminf _{\epsilon \rightarrow 0} \lambda\right) \Psi_{\infty} .
$$

The equation (4.17) has no bounded solution if $\lim \inf \lambda<0$. Hence $\lim \inf \lambda=0$. Then the only bounded solutions are scalar multiples of $H^{\prime}$. Hence $\Psi_{\infty}=c H^{\prime}$ for some $c \neq 0$. But this contradicts (4.15).

We are now ready to show that $w_{\xi}+\phi_{\xi}$ locally minimizes $I$ in the $\xi$-fiber.

Lemma 4.2 There exists an non-empty open $\mathcal{N}_{\xi} \subset \mathcal{P}_{\xi}$ containing 0 such that for every $\psi \in \mathcal{N}_{\xi}$, $\psi \neq 0, I\left(w_{\xi}+\phi_{\xi}\right)<I\left(w_{\xi}+\phi_{\xi}+\psi\right)$.

Proof. We again denote $w_{\xi}+\phi_{\xi}$ by $g_{\xi}$. Let $\psi \in \mathcal{P}_{\xi}$ and expand

$$
I\left(g_{\xi}+\psi\right)=I\left(g_{\xi}\right)+\int_{0}^{1} S\left(g_{\xi}\right) \psi+\frac{1}{2} \int_{0}^{1}\left[\epsilon^{2}\left(\psi^{\prime}\right)^{2}+f^{\prime}\left(g_{\xi}\right) \psi^{2}+\sigma\left|(-\Delta)^{-1 / 2} \psi\right|^{2}\right]+\frac{1}{6} \int_{0}^{1} f^{\prime \prime}(. .) \psi^{3} .
$$


Because $\pi_{\xi} \circ S\left(g_{\xi}\right)=0$ and $\psi \perp h_{j}$, we deduce

$$
I\left(g_{\xi}+\psi\right)=I\left(g_{\xi}\right)+\frac{1}{2} \int_{0}^{1}\left[\epsilon^{2}\left(\psi^{\prime}\right)^{2}+f^{\prime}\left(g_{\xi}\right) \psi^{2}+\sigma\left|(-\Delta)^{-1 / 2} \psi\right|^{2}\right] d x+O(\|\psi\|)\|\psi\|_{2}^{2}
$$

which by (4.2) implies

$$
I\left(g_{\xi}+\psi\right) \geq I\left(g_{\xi}\right)+\left(\frac{\lambda}{2}-O(\|\psi\|)\right)\|\psi\|_{2}^{2}
$$

where $C$ is independent of $\epsilon$. We define a non-empty open subset

$$
\mathcal{N}_{\xi}=\left\{\psi \in \mathcal{P}_{\xi}:\|\psi\|<C_{1}\right\}
$$

of $\mathcal{P}_{\xi}$ for some $C_{1}>0$. If we take $C_{1}$ to be sufficiently small (but independent of $\epsilon$ ), then (4.20) and Lemma 4.1 imply that there exists $C_{2}>0$, independent of $\epsilon$, so that

$$
I\left(g_{\xi}+\psi\right) \geq I\left(g_{\xi}\right)+C_{2}\|\psi\|_{2}^{2}, \text { for all } \psi \in \mathcal{N}_{\xi} .
$$

Lemma 4.2 follows from (4.22).

\section{The reduced problem}

In this section we find a particular $\tilde{\xi}$ so that $\tilde{\xi}$ locally minimizes $I\left(w_{\xi}+\phi_{\xi}\right)$ with respect to $\xi$ and $S\left(w_{\tilde{\xi}}+\phi_{\tilde{\xi}}\right)=0$. Let

$$
J(\zeta, l)=I\left(w_{\xi}+\phi_{\xi}\right)
$$

where $\zeta$ and $l$ are related to $\xi$ via (2.3).

Lemma 5.1 For $\xi \in \Lambda$ we have

$$
J(\zeta, l)=\epsilon\left[2 N \tau+4 f^{\prime}(0) Q(\zeta, l)\right]+O\left(\epsilon^{3 / 2}\right)
$$

uniformly with respect to $(\zeta, l)$. Here $Q$ is an $\epsilon$-independent function

$$
Q(\zeta, l)=\frac{1}{2} \sum_{k, j=1}^{N} l_{k} l_{j} G_{D}\left(\zeta_{k}, \zeta_{j}\right)-\frac{a_{0}}{2} \sum_{j=1}^{N} l_{j}+\frac{a_{0}^{2}}{8}
$$

for $(\zeta, l)$ that satisfies $0<\zeta_{1}<\zeta_{2}<\ldots<\zeta_{N}<1, l_{j}>0$, for $j=1,2, \ldots, 2 N$.

Proof. We expand the energy of $w_{\xi}+\phi_{\xi}$ to find

$$
J(\zeta, l)=I\left(w_{\xi}\right)+\int_{0}^{1} S\left(w_{\xi}\right) \phi_{\xi}+\frac{1}{2} \int_{0}^{1}\left[\epsilon^{2}\left|\phi_{\xi}^{\prime}\right|^{2}+f^{\prime}\left(w_{\xi}\right) \phi_{\xi}^{2}+\sigma\left|(-\Delta)^{-1 / 2} \phi_{\xi}\right|^{2}\right] d x+O\left(\epsilon^{3 / 2}\right) .
$$

The equation $\pi_{\xi} \circ S\left(w_{\xi}+\phi_{\xi}\right)=0$ implies that

$$
-\epsilon^{2} \phi_{\xi}^{\prime \prime}+f^{\prime}\left(w_{\xi}\right) \phi_{\xi}-\overline{f^{\prime}\left(w_{\xi}\right) \phi_{\xi}}+\sigma(-\Delta)^{-1} \phi_{\xi}+S\left(w_{\xi}\right)+O\left(\left\|\phi_{\xi}\right\|^{2}\right)=\sum_{j=1}^{2 N} \beta_{j} h_{j}
$$


for some $\beta_{j} \in \mathbf{R}$. Multiply (5.5) by $\phi_{\xi}$ and integrate to find

$$
\int_{0}^{1}\left[\epsilon^{2}\left|\phi_{\xi}^{\prime}\right|^{2}+f^{\prime}\left(w_{\xi}\right) \phi_{\xi}^{2}+\sigma\left|(-\Delta)^{-1 / 2} \phi_{\xi}\right|^{2}\right] d x+\int_{0}^{1} S\left(w_{\xi}\right) \phi_{\xi} d x+O\left(\epsilon^{3 / 2}\right)=0 .
$$

Substituting (5.6) to (5.4) we deduce

$$
J(\zeta, l)=I\left(w_{\xi}\right)+\frac{1}{2} \int_{0}^{1} S\left(w_{\xi}\right) \phi_{\xi} d x+O\left(\epsilon^{3 / 2}\right)
$$

By Lemma 2.2 we obtain

$$
\int_{0}^{1} S\left(w_{\xi}\right) \phi_{\xi} d x=\int_{0}^{1}\left(f^{\prime}\left(w_{\xi, 1}\right)-f^{\prime}(0)\right) w_{\xi, 2} \phi_{\xi} d x+O\left(\epsilon^{3 / 2}\right)=O\left(\epsilon^{3 / 2}\right) .
$$

To see the last equation note that only on intervals of size $\epsilon$ near $\xi_{j}, f^{\prime}\left(w_{\xi, 1}\right)-f^{\prime}(0)$ is of order 1 . Elsewhere $f^{\prime}\left(w_{\xi, 1}\right)-f^{\prime}(0)=O(\epsilon)$. Now (5.7) becomes

$$
J(\zeta, l)=I\left(w_{\xi}\right)+O\left(\epsilon^{3 / 2}\right) .
$$

Therefore we turn our attention to $I\left(w_{\xi}\right)$. Note that

$$
\begin{aligned}
I\left(w_{\xi}\right)= & \frac{\epsilon^{2}}{2} \int_{0}^{1}\left|w_{\xi, 1}^{\prime}\right|^{2} d x+\epsilon^{2} \int_{0}^{1} w_{\xi, 1}^{\prime} w_{\xi, 2}^{\prime} d x \\
& +\int_{0}^{1} W\left(w_{\xi, 1}\right) d x+\int_{0}^{1} f\left(w_{\xi, 1}\right) w_{\xi, 2} d x+\frac{1}{2} \int_{0}^{1} f^{\prime}\left(w_{\xi, 1}\right) w_{\xi, 2}^{2} d x \\
& +\frac{\sigma}{2} \int_{0}^{1}\left|(-\Delta)^{-1 / 2}\left(w_{\xi, 1}+w_{\xi, 2}-a\right)\right|^{2} d x+O\left(\epsilon^{3 / 2}\right) \\
= & \frac{\epsilon^{2}}{2} \int_{0}^{1}\left|w_{\xi, 1}^{\prime}\right|^{2} d x+\int_{0}^{1} W\left(w_{\xi, 1}\right) d x \\
& +\epsilon^{2} \int_{0}^{1} w_{\xi, 1}^{\prime} w_{\xi, 2}^{\prime} d x+\int_{0}^{1} f\left(w_{\xi, 1}\right) w_{\xi, 2} d x \\
& +\frac{1}{2} \int_{0}^{1} f^{\prime}\left(w_{\xi, 1}\right) w_{\xi, 2}^{2} d x+\frac{\sigma}{2} \int_{0}^{1}\left|(-\Delta)^{-1 / 2}\left(w_{\xi, 1}+w_{\xi, 2}-a\right)\right|^{2} d x+O\left(\epsilon^{3 / 2}\right) \\
= & 2 N \tau \epsilon+\frac{1}{2} \int_{0}^{1} f^{\prime}\left(w_{\xi, 1}\right) w_{\xi, 2}^{2} \\
& +\frac{\sigma}{2} \int_{0}^{1}\left(w_{\xi, 1}+w_{\xi, 2}-a\right)(-\Delta)^{-1}\left(w_{\xi, 1}+w_{\xi, 2}-a\right)+O\left(\epsilon^{3 / 2}\right) \\
= & 2 N \tau \epsilon+\frac{1}{2} \int_{0}^{1} f^{\prime}\left(w_{\xi, 1}\right) w_{\xi, 2}^{2}-\frac{f^{\prime}(0)}{2} \int_{0}^{1}\left(w_{\xi, 1}+w_{\xi, 2}-a\right) w_{\xi, 2}+O\left(\epsilon^{3 / 2}\right) \\
= & 2 N \tau \epsilon+\frac{1}{2} \int_{0}^{1}\left(f^{\prime}\left(w_{\xi, 1}\right)-f^{\prime}(0)\right) w_{\xi, 2}^{2}-\frac{f^{\prime}(0)}{2} \int_{0}^{1}\left(w_{\xi, 1}-a\right) w_{\xi, 2}+O\left(\epsilon^{3 / 2}\right) \\
= & 2 N \tau \epsilon-\frac{f^{\prime}(0)}{2} \int_{0}^{1}\left(w_{\xi, 1}-a\right) w_{\xi, 2} d x+O\left(\epsilon^{3 / 2}\right)
\end{aligned}
$$




$$
\begin{aligned}
= & 2 N \tau \epsilon-\frac{f^{\prime}(0) \epsilon}{2} \int_{0}^{1}\left(\frac{w_{\xi, 1}}{\epsilon^{1 / 2}}-a_{0}\right)\left(a_{0}-\sum_{j=1}^{N} 2 l_{j} G_{D}\left(x, \zeta_{j}\right)\right) d x+O\left(\epsilon^{3 / 2}\right) \\
= & 2 N \tau \epsilon-\frac{f^{\prime}(0) \epsilon}{2}\left[\int_{0}^{1} \frac{w_{\xi, 1}}{\epsilon^{1 / 2}} a_{0} d x-2 \int_{0}^{1} \frac{w_{\xi, 1}}{\epsilon^{1 / 2}}\left(\sum_{j=1}^{N} l_{j} G_{D}\left(x, \zeta_{j}\right)\right) d x\right. \\
& \left.-a_{0}^{2}+2 a_{0} \int_{0}^{1}\left(\sum_{j=1}^{N} l_{j} G_{D}\left(x, \zeta_{j}\right)\right) d x\right]+O\left(\epsilon^{3 / 2}\right) \\
= & 2 N \tau \epsilon-\frac{f^{\prime}(0) \epsilon}{2}\left[2 a_{0} \sum_{j=1}^{N} l_{j}-4 \sum_{k, j=1}^{N} l_{k} l_{j} G_{D}\left(\zeta_{k}, \zeta_{j}\right)-a_{0}^{2}+2 a_{0} \sum_{j=1}^{N} l_{j}\right]+O\left(\epsilon^{3 / 2}\right)(5.2) \\
= & 2 N \tau \epsilon+4 f^{\prime}(0) \epsilon\left[\frac{1}{2} \sum_{k, j=1}^{N} l_{k} l_{j} G_{D}\left(\zeta_{k}, \zeta_{j}\right)-\frac{a_{0}}{2} \sum_{j=1}^{N} l_{j}+\frac{a_{0}^{2}}{8}\right]+O\left(\epsilon^{3 / 2}\right)
\end{aligned}
$$

This proves the lemma.

Lemma 5.2 $Q$ has a unique critical point $\left(\zeta^{0}, l^{0}\right)$ where

$$
\zeta_{1}^{0}=\frac{1}{2 N}, \zeta_{2}^{0}=\frac{3}{2 N}, \zeta_{3}^{0}=\frac{5}{2 N}, \ldots, \zeta_{j}^{0}=\frac{2 j-1}{2 N}, \ldots, \zeta_{N}^{0}=\frac{2 N-1}{2 N}
$$

and

$$
l_{j}^{0}=a_{0} \sqrt{D}\left(\operatorname{coth} \frac{1}{N \sqrt{D}}-\operatorname{csch} \frac{1}{N \sqrt{D}}\right), j=1,2, \ldots, N .
$$

Proof. For any $(\zeta, l)$ we define a $C^{0,1}[0,1]$ function $p$ by

$$
p(x)=\sum_{k=1}^{N} G_{D}\left(x, \zeta_{k}\right) l_{k} .
$$

Calculations show that

$$
\begin{aligned}
\frac{\partial Q}{\partial \zeta_{j}} & =\frac{l_{j}}{2}\left(p^{\prime}\left(\zeta_{j}-\right)+p^{\prime}\left(\zeta_{j}+\right)\right), \\
\frac{\partial Q}{\partial l_{j}} & =\sum_{k=1}^{N} G_{D}\left(\zeta_{j}, \zeta_{k}\right) l_{k}-\frac{a_{0}}{2}=p\left(\zeta_{j}\right)-\frac{a_{0}}{2} .
\end{aligned}
$$

Now we assume that $(\zeta, l)$ is a critical point of $Q$. Then (5.28) and (5.29) imply that

$$
\begin{aligned}
p^{\prime}\left(\zeta_{j}-\right)+p^{\prime}\left(\zeta_{j}+\right) & =0, \\
p\left(\zeta_{j}\right) & =\frac{a_{0}}{2} .
\end{aligned}
$$

Since $p$ satisfies the linear differential equation

$$
-D p^{\prime \prime}+p=0 \text {, in }(0,1) \backslash\left\{\zeta_{1}, \zeta_{2}, \ldots, \zeta_{N}\right\} \text {, and } p^{\prime}(0)=p^{\prime}(1)=0,
$$


(5.30) and (5.31) imply that

$$
\zeta_{1}=\frac{1}{2 N}, \zeta_{2}=\frac{3}{2 N}, \zeta_{3}=\frac{5}{2 N}, \ldots, \zeta_{j}=\frac{2 j-1}{2 N}, \ldots, \zeta_{N}=\frac{2 N-1}{2 N} .
$$

At a critical point $(\zeta, l),(5.29)$ implies that $l$ satisfies the linear system

$$
\left[\begin{array}{rrrr}
G_{D}\left(\zeta_{1}, \zeta_{1}\right) & G_{D}\left(\zeta_{1}, \zeta_{2}\right) & \ldots & G_{D}\left(\zeta_{1}, \zeta_{N}\right) \\
G_{D}\left(\zeta_{2}, \zeta_{1}\right) & G_{D}\left(\zeta_{2}, \zeta_{2}\right) & \ldots & G_{D}\left(\zeta_{2}, \zeta_{N}\right) \\
\ldots & & & \\
G_{D}\left(\zeta_{N}, \zeta_{1}\right) & G_{D}\left(\zeta_{N}, \zeta_{2}\right) & \ldots & G_{D}\left(\zeta_{N}, \zeta_{N}\right)
\end{array}\right]\left[\begin{array}{c}
l_{1} \\
l_{2} \\
\ldots \\
l_{N}
\end{array}\right]=\frac{1}{2}\left[\begin{array}{c}
a_{0} \\
a_{0} \\
\ldots \\
a_{0}
\end{array}\right]
$$

It is shown in the appendix that at (5.33) the symmetric $N \times N$ matrix $\left[G_{D}\left(\zeta_{j}, \zeta_{k}\right)\right]$ is non-singular and

$$
\sum_{k=1}^{N} G_{D}\left(\zeta_{j}, \zeta_{k}\right)=\frac{1}{2 \sqrt{D}} \frac{1}{\operatorname{coth}(1 /(N \sqrt{D}))-\operatorname{csch}(1 /(N \sqrt{D}))} .
$$

Note that the right side of (5.35) is independent of $j$. Therefore the unique solution to (5.34) is

$$
l_{j}=a_{0} \sqrt{D}\left(\operatorname{coth} \frac{1}{N \sqrt{D}}-\operatorname{csch} \frac{1}{N \sqrt{D}}\right) .
$$

This proves the lemma.

Lemma 5.3 The second derivative matrix of $Q$ at $\left(\zeta^{0}, l^{0}\right)$ is positive definite.

Proof. We calculate the second derivative of $Q$ at $\left(\zeta^{0}, l^{0}\right)$. First

$$
\frac{\partial^{2} Q}{\partial l_{j} \partial l_{k}}\left(\zeta^{0}, l^{0}\right)=G_{D}\left(\zeta_{j}^{0}, \zeta_{k}^{0}\right)
$$

We define a matrix $\mathbf{A}$ whose $i k$-entry $a_{j k}$ is given by (5.37).

Next we write $G_{D}$ as

$$
G_{D}(x, y)=\Gamma(|x-y|)+R(x, y)
$$

where $\Gamma$ is the fundamental solution and $R$ is the smooth regular part:

$$
\Gamma(z)=\frac{1}{2 \sqrt{D}} \exp \left(-\frac{z}{\sqrt{D}}\right), \quad R(x, y)=\frac{\cosh \frac{x+y-1}{\sqrt{D}}+\exp \left(-\frac{1}{\sqrt{D}}\right) \cosh \frac{x-y}{\sqrt{D}}}{2 \sqrt{D} \sinh \frac{1}{\sqrt{D}}} .
$$

Then we calculate

$$
\begin{aligned}
\frac{\partial Q}{\partial \zeta_{j}} & =\frac{1}{2} \frac{\partial}{\partial \zeta_{j}}\left[G_{D}\left(\zeta_{j}, \zeta_{j}\right) l_{j}^{2}\right]+\frac{\partial}{\partial \zeta_{j}}\left[\sum_{k \neq j} G_{D}\left(\zeta_{j}, \zeta_{k}\right) l_{j} l_{k}\right] \\
& =R_{1}\left(\zeta_{j}, \zeta_{j}\right) l_{j}^{2}+l_{j} \sum_{k \neq j}\left[\Gamma^{\prime}\left(\left|\zeta_{j}-\zeta_{k}\right|\right)( \pm 1)+R_{1}\left(\zeta_{j}, \zeta_{k}\right)\right] l_{k}
\end{aligned}
$$

where $\pm 1=1$ if $\zeta_{j}-\zeta_{k}>0$ and $\pm=-1$ if $\zeta_{j}-\zeta_{k}<0$. Let

$$
\iota=l_{1}^{0}=l_{2}^{0}=\ldots=l_{N}^{0}=a_{0} \sqrt{D}\left(\operatorname{coth} \frac{1}{N \sqrt{D}}-\operatorname{csch} \frac{1}{N \sqrt{D}}\right) .
$$


Then if $k \neq j$

$$
\frac{\partial^{2} Q\left(\zeta^{0}, l^{0}\right)}{\partial \zeta_{j} \partial l_{k}}=\iota\left(\Gamma^{\prime}\left(\left|\zeta_{j}^{0}-\zeta_{k}^{0}\right|\right)( \pm 1)+R_{1}\left(\zeta_{j}^{0}, \zeta_{k}^{0}\right)\right), j \neq k
$$

If $k=j$,

$$
\frac{\partial^{2} Q\left(\zeta^{0}, l^{0}\right)}{\partial \zeta_{j} \partial l_{j}}=2 \iota R_{1}\left(\zeta_{j}^{0}, \zeta_{j}^{0}\right)+\iota \sum_{k \neq j}\left(\Gamma^{\prime}\left(\left|\zeta_{j}^{0}-\zeta_{k}^{0}\right|\right)( \pm 1)+R_{1}\left(\zeta_{j}^{0}, \zeta_{k}^{0}\right)\right) .
$$

We define a matrix $\mathbf{B}$ whose $j k$-entry $b_{j k}$ is given by (5.43) and (5.44).

If $k \neq j$,

$$
\frac{\partial^{2} Q\left(\zeta^{0}, l^{0}\right)}{\partial \zeta_{j} \partial \zeta_{k}}=\iota^{2}\left(-\Gamma^{\prime \prime}\left(\left|\zeta_{j}^{0}-\zeta_{k}^{0}\right|\right)+R_{12}\left(\zeta_{j}^{0}, \zeta_{k}^{0}\right)\right)
$$

If $k=j$,

$$
\frac{\partial^{2} Q\left(\zeta^{0}, l^{0}\right)}{\partial \zeta_{j}^{2}}=\iota^{2}\left(R_{11}\left(\zeta_{j}^{0}, \zeta_{j}^{0}\right)+R_{12}\left(\zeta_{j}^{0}, \zeta_{j}^{0}\right)\right)+\iota^{2} \sum_{k \neq j}\left[\Gamma^{\prime \prime}\left(\left|\zeta_{j}^{0}-\zeta_{k}^{0}\right|\right)+R_{11}\left(\zeta_{j}^{0}, \zeta_{k}^{0}\right)\right]
$$

Using the the equation that $G_{D}$ solves we may simply the second part on the right side and obtain

$$
\frac{\partial^{2} Q\left(\zeta^{0}, l^{0}\right)}{\partial \zeta_{j}^{2}}=\iota^{2}\left(-\Gamma^{\prime \prime}(0)+R_{12}\left(\zeta_{j}^{0}, \zeta_{j}^{0}\right)\right)+\frac{\iota^{2}}{D} \sum_{k=1}^{N} G_{D}\left(\zeta_{j}^{0}, \zeta_{k}^{0}\right)
$$

We define a matrix $\mathbf{C}$ whose $j k$-entry $c_{j k}$ is given by (5.45) and (5.47).

The second derivative of $Q$ at $\left(\zeta^{0}, l^{0}\right)$ is the symmetric matrix

$$
\mathbf{H}=\left[\begin{array}{ll}
\mathbf{A} & \mathbf{B}^{T} \\
\mathbf{B} & \mathbf{C}
\end{array}\right]
$$

It turns out that the matrices $\mathbf{A}, \mathbf{B}$, and $\mathbf{C}$ also appear in the stability analysis of multiple spike solutions to the Gierer-Meinhardt system. See Iron, Ward and Wei [8], and Wei and Winter [27]. By using the spectral information of $\mathbf{A}, \mathbf{B}$, and $\mathbf{C}$ we can show that the matrix $\mathbf{H}$ is positive definite. The argument is a bit long. We leave the complete proof to the appendix.

Proof of Theorem 1.1. Let us denote $w_{\xi}$ and $\phi_{\xi}$ by $w_{\zeta, l}$ and $\phi_{\zeta, l}$ respectively where $\xi$ is related to $\zeta$ and $l$ via (2.3). In $\Lambda$, according to Lemma 5.1, $\epsilon^{-1} J$ uniformly converges to $2 N \tau+4 f^{\prime}(0) Q$ as $\epsilon \rightarrow 0$. Lemmas 5.2 and 5.3 imply that $\left(\zeta^{0}, l^{0}\right)$ is a strict local minimizer of $2 N \tau+4 f^{\prime}(0) Q$. Our choice of $\delta$ in the definition (2.4) of $\Lambda$ ensures that $\left(\zeta^{0}, l^{0}\right) \in \Lambda$. Near $\left(\zeta^{0}, l^{0}\right)$ there exists a local minimizer $(\tilde{\zeta}, \tilde{l})$ of $J$ when $\epsilon$ is sufficiently small. Moreover $(\tilde{\zeta}, \tilde{l}) \rightarrow\left(\zeta^{0}, l^{0}\right)$ as $\epsilon \rightarrow 0$. It is standard to prove that $S\left(w_{\tilde{\zeta}, \tilde{l}}+\phi_{\tilde{\zeta}, \tilde{l}}\right)=0$. The detailed argument may be found in papers like [6, 23]. Lemma 4.2 states that for each $(\zeta, l), w_{\zeta, l}+\phi_{\zeta, l}$ locally minimizes $I$ in the fiber $w_{\zeta, l}+\mathcal{P}_{\zeta, l}$. Then $w_{\tilde{\zeta}, \tilde{l}}+\phi_{\tilde{\zeta}, \tilde{l}}$ locally minimizes $I$ in $\mathcal{A}$.

The energy estimate of the local minimizer is derived from Lemma 5.1, and the values of $\zeta^{0}$ and $l^{0}$ are given in Lemma 5.2, and (5.35). 


\section{Discussion}

The size of each droplet of the local minimizer constructed in Theorem 1.1 is of order $\epsilon^{1 / 2}$ and the free energy of the local minimizer is of order $\epsilon$. Interestingly the free energy of the constant $a$, which is also a local minimizer, is

$$
\frac{f^{\prime}(0) a_{0}^{2}}{2} \epsilon+O\left(\epsilon^{3 / 2}\right),
$$

again of order $\epsilon$. This suggests that if $a \ll \epsilon^{1 / 2}$, any droplet solution, if it exists, will have free energy much larger than the free energy of the constant $a$. So our parameter range (1.7) may well be an important threshold.

If in Theorem 1.1 we expand

$$
\frac{\operatorname{coth} \beta-\operatorname{csch} \beta}{\beta}=\frac{1}{2}-\frac{\beta^{2}}{24}+\ldots
$$

for small $\beta$, i.e. large $N$, we find that the free energy of the $N$ droplet solution is approximately

$$
\epsilon\left(2 N \tau+\frac{a_{0}^{2} \sigma}{24 N^{2}}\right) .
$$

The last expression is minimized at

$$
N_{\text {opt }}=\left(\frac{a_{0}^{2} \sigma}{24 \tau}\right)^{1 / 3}
$$

with respect to $N$. This $N_{o p t}$ gives the optimal number of droplets.

Another interesting consequence of our work concerns the distribution of the $A$-monomers. The area under each droplet of an $N$-droplet local minimizer, by (5.36), is

$$
\frac{2 a_{0}}{N \beta}(\operatorname{coth} \beta-\operatorname{csch} \beta) \epsilon^{1 / 2}+o\left(\epsilon^{1 / 2}\right) .
$$

Taking all the droplets into consideration, the area under all the droplets is

$$
\frac{2 a_{0}}{\beta}(\operatorname{coth} \beta-\operatorname{csch} \beta) \epsilon^{1 / 2}+o\left(\epsilon^{1 / 2}\right) .
$$

On the other hand the total area under the graph of the local minimizer is $a=a_{0} \epsilon^{1 / 2}$. Since (6.2) implies that

$$
\frac{2 a_{0}}{\beta}(\operatorname{coth} \beta-\operatorname{csch} \beta)<a_{0},
$$

we discover that a significant portion of the area $a_{0} \epsilon^{1 / 2}$ is under the part where the graph is close to 0 . In other words the $A$-monomers form droplets and at the same time spread through the entire sample with the $B$-monomers.

\section{Appendix}

Here we show that $G_{D}\left(\zeta_{j}^{0}, \zeta_{k}^{0}\right)$ is non-singular, verify (5.35), and prove that all the eigenvalues of $\mathbf{H}$ are positive. 
Arguing as in [19, Section 7] we find that the inverse matrix of $\mathbf{A}$ has the tridiagonal form

$$
\mathbf{A}^{-1}=\sqrt{D}\left[\begin{array}{cccccc}
2 \operatorname{coth} \beta-\operatorname{csch} \beta & -\operatorname{csch} \beta & 0 & 0 & \ldots & 0 \\
-\operatorname{csch} \beta & 2 \operatorname{coth} \beta & -\operatorname{csch} \beta & 0 & \ldots & 0 \\
0 & -\operatorname{csch} \beta & 2 \operatorname{coth} \beta & -\operatorname{csch} \beta & \ldots & 0 \\
\ldots & & & & & \\
0 & 0 & 0 & 0 & \ldots & 2 \operatorname{coth} \beta-\operatorname{csch} \beta
\end{array}\right]
$$

where

One rewrites (A.1) as

$$
\beta=\frac{1}{N \sqrt{D}}
$$

$$
\mathbf{A}^{-1}=-\sqrt{D} \operatorname{csch} \beta\left[\begin{array}{rrrrrr}
1 & 1 & 0 & 0 & \ldots & 0 \\
1 & 0 & 1 & 0 & \ldots & 0 \\
0 & 1 & 0 & 1 & \ldots & 0 \\
\ldots & & & & & \\
0 & 0 & 0 & 0 & \ldots & 1
\end{array}\right]+2 \sqrt{D} \operatorname{coth} \beta \mathbf{I}
$$

where $\mathbf{I}$ is the $N \times N$ identity matrix. One just needs to diagonalized the first matrix on the right side of (A.3). It turns out that $\mathbf{A}^{-1}$ is diagonalized by the orthogonal matrix of eigenvectors

$$
\mathbf{P}=\sqrt{\frac{2}{N}}\left[\begin{array}{cccc}
\frac{1}{\sqrt{2}} & \cos \left(\frac{\pi}{N}(2-1)\left(1-\frac{1}{2}\right)\right) & \ldots & \cos \left(\frac{\pi}{N}(N-1)\left(1-\frac{1}{2}\right)\right) \\
\frac{1}{\sqrt{2}} & \cos \left(\frac{\pi}{N}(2-1)\left(2-\frac{1}{2}\right)\right) & \ldots & \cos \left(\frac{\pi}{N}(N-1)\left(2-\frac{1}{2}\right)\right) \\
\ldots & & & \\
\frac{1}{\sqrt{2}} & \cos \left(\frac{\pi}{N}(2-1)\left(N-\frac{1}{2}\right)\right) & \ldots & \cos \left(\frac{\pi}{N}(N-1)\left(N-\frac{1}{2}\right)\right)
\end{array}\right]
$$

and that

$$
\mathbf{P}^{T} \mathbf{A}^{-1} \mathbf{P}=-\sqrt{D} \operatorname{csch} \beta\left[\begin{array}{llll}
\lambda_{1} & 0 & \ldots & 0 \\
0 & \lambda_{2} & \ldots & 0 \\
\ldots & & & \\
0 & 0 & \ldots & \lambda_{N}
\end{array}\right]+2 \sqrt{D} \operatorname{coth} \beta \mathbf{I}
$$

where

$$
\lambda_{j}=2 \cos \frac{\pi(j-1)}{N}, j=1,2, \ldots, N .
$$

Therefore

$$
\mathbf{P}^{T} \mathbf{A P}=\left[\begin{array}{llll}
a_{1} & 0 & \ldots & 0 \\
0 & a_{2} & \ldots & 0 \\
\ldots & & & \\
0 & 0 & \ldots & a_{N}
\end{array}\right]
$$

where the eigenvalues of $\mathbf{A}$ are

$$
a_{j}=\frac{1}{2 \sqrt{D}} \frac{1}{\operatorname{coth} \beta-\operatorname{csch} \beta \cos \frac{\pi(j-1)}{N}}>0, j=1,2, \ldots, N .
$$

Hence all the eigenvalues of $G_{D}\left(\zeta_{j}^{0}, \zeta_{k}^{0}\right)$ are positive and

$$
\sum_{k=1}^{N} G_{D}\left(\zeta_{j}^{0}, \zeta_{k}^{0}\right)=a_{1}=\frac{1}{2 \sqrt{D}} \frac{1}{\operatorname{coth} \beta-\operatorname{csch} \beta}
$$


is independent of $j$, proving (5.35).

The formula (A.9) also allows us to simplify $\mathbf{C}$ to

$$
\mathbf{C}=-\iota^{2} \mathbf{D}+\frac{\iota^{2}}{2 D^{3 / 2}(\operatorname{coth} \beta-\operatorname{csch} \beta)} \mathbf{I}
$$

where the $j k$ entry of $\mathbf{D}$ is

$$
d_{j k}=\Gamma^{\prime \prime}\left(\left|\zeta_{j}^{0}-\zeta_{k}^{0}\right|\right)-R_{12}\left(\zeta_{j}^{0}, \zeta_{k}^{0}\right)
$$

However from (5.39) we find that

$$
\Gamma^{\prime \prime}(|x-y|)-R_{12}(x, y)
$$

is the Green function of

$$
-D z^{\prime \prime}+z=\delta(\cdot-y), z(0)=z(1)=1
$$

divided by $D$. Argument similar to $[19$, Section 7$]$ shows that

$$
\mathbf{D}^{-1}=D^{3 / 2}\left[\begin{array}{cccccc}
2 \operatorname{coth} \beta+\operatorname{csch} \beta & -\operatorname{csch} \beta & 0 & 0 & \ldots & 0 \\
-\operatorname{csch} \beta & 2 \operatorname{coth} \beta & -\operatorname{csch} \beta & 0 & \ldots & 0 \\
0 & -\operatorname{csch} \beta & 2 \operatorname{coth} \beta & -\operatorname{csch} \beta & \ldots & 0 \\
\ldots & 0 & 0 & 0 & \ldots & 2 \operatorname{coth} \beta+\operatorname{csch} \beta
\end{array}\right]
$$

which is diagonalized by

$$
\mathbf{Q}=\sqrt{\frac{2}{N}}\left[\begin{array}{rrrr}
\frac{-1}{\sqrt{2}} & \sin \left(\frac{\pi}{N}(2-1)\left(1-\frac{1}{2}\right)\right) & \ldots & \sin \left(\frac{\pi}{N}(N-1)\left(1-\frac{1}{2}\right)\right) \\
\frac{1}{\sqrt{2}} & \sin \left(\frac{\pi}{N}(2-1)\left(2-\frac{1}{2}\right)\right) & \ldots & \sin \left(\frac{\pi}{N}(N-1)\left(2-\frac{1}{2}\right)\right) \\
\ldots & & & \\
\frac{(-1)^{N}}{\sqrt{2}} & \sin \left(\frac{\pi}{N}(2-1)\left(N-\frac{1}{2}\right)\right) & \ldots & \sin \left(\frac{\pi}{N}(N-1)\left(N-\frac{1}{2}\right)\right)
\end{array}\right]
$$

Then

$$
\mathbf{Q}^{T} \mathbf{D}^{-1} \mathbf{Q}=-D^{3 / 2} \operatorname{csch} \beta\left[\begin{array}{llll}
\mu_{1} & 0 & \ldots & 0 \\
0 & \mu_{2} & \ldots & 0 \\
\ldots & & & \\
0 & 0 & \ldots & \mu_{N}
\end{array}\right]+2 D^{3 / 2} \operatorname{coth} \beta \mathbf{I}
$$

where the $\mu_{j}$ 's are

$$
\mu_{1}=-2, \mu_{j}=2 \cos \frac{\pi(j-1)}{N}, j=2,3, \ldots, N
$$

Hence

$$
\mathbf{Q}^{T} \mathbf{C Q}=\left[\begin{array}{llll}
c_{1} & 0 & \ldots & 0 \\
0 & c_{2} & \ldots & 0 \\
\ldots & & & \\
0 & 0 & \ldots & c_{N}
\end{array}\right]
$$

where

$$
\begin{aligned}
& c_{1}=\frac{\iota^{2}}{2 D^{3 / 2}}\left(\frac{1}{\operatorname{coth} \beta-\operatorname{csch} \beta}-\frac{1}{\operatorname{coth} \beta+\operatorname{csch} \beta}\right)>0, \\
& c_{j}=\frac{\iota^{2}}{2 D^{3 / 2}}\left(\frac{1}{\operatorname{coth} \beta-\operatorname{csch} \beta}-\frac{1}{\operatorname{coth} \beta-\operatorname{csch} \beta \cos \frac{\pi(j-1)}{N}}\right)>0, j=2,3, \ldots, N .
\end{aligned}
$$


We will use

$$
\left[\begin{array}{cc}
\mathbf{P} & \mathbf{0} \\
\mathbf{0} & \mathbf{Q}
\end{array}\right]
$$

to diagonalize $\mathbf{H}$ to $2 \times 2$ blocks. We must compute $\mathbf{Q}^{T} \mathbf{B P}$. Let

$$
\mathbf{B}=\iota(\mathbf{E}+\mathbf{F})
$$

where the $j k$ entry $e_{j k}$ of $\mathbf{E}$ is

$$
e_{j k}=\left\{\begin{array}{rll}
\Gamma^{\prime}\left(\left|\zeta_{j}^{0}-\zeta_{k}^{0}\right|\right)( \pm 1)+R_{1}\left(\zeta_{j}^{0}, \zeta_{k}^{0}\right) & \text { if } j \neq k \\
R_{1}\left(\zeta_{j}^{0}, \zeta_{j}^{0}\right) & \text { if } \quad j=k
\end{array}\right.
$$

and $\mathbf{F}$ is diagonal whose $j j$ entry is

$$
f_{j j}=R_{1}\left(\zeta_{j}^{0}, \zeta_{j}^{0}\right)+\sum_{k \neq j}\left(\Gamma^{\prime}\left(\left|\zeta_{j}^{0}-\zeta_{k}^{0}\right|\right)( \pm 1)+R_{1}\left(\zeta_{j}^{0}, \zeta_{k}^{0}\right)\right)
$$

Recall that $\pm 1=-1$ if $\zeta_{j}^{0}<\zeta_{k}^{0}$ and $\pm 1=1$ if $\zeta_{j}^{0}>\zeta_{k}^{0}$.

To study $\mathbf{Q}^{T} \mathbf{E P}$, we write $\mathbf{E}$ as $\mathbf{M A}$ where, after some calculations, we find that

$$
\mathbf{M}=\mathbf{E A}^{-1}=\frac{\operatorname{csch} \beta}{2 \sqrt{D}}\left[\begin{array}{rrrrrr}
-1 & 1 & 0 & 0 & \ldots & 0 \\
-1 & 0 & 1 & 0 & \ldots & 0 \\
0 & -1 & 0 & 1 & \ldots & 0 \\
\ldots & & & & & \\
0 & 0 & 0 & 0 & \ldots & -1
\end{array}\right]
$$

Then

$$
\mathbf{Q}^{T} \mathbf{E P}=\left(\mathbf{M}^{T} \mathbf{Q}\right)^{T}(\mathbf{A P}) .
$$

If we denote the columns of $\mathbf{P}$ by $\mathbf{p}_{1}, \mathbf{p}_{2}, \ldots, \mathbf{p}_{N}$, then

$$
\mathbf{A P}=\left[a_{1} \mathbf{p}_{1}, a_{2} \mathbf{p}_{2}, \ldots, a_{N} \mathbf{p}_{N}\right],
$$

and

$$
\mathbf{M}^{T} \mathbf{Q}=-\frac{\operatorname{csch} \beta}{\sqrt{D}}\left[0 \mathbf{p}_{1}, \sin \frac{\pi}{N} \mathbf{p}_{2}, \ldots, \sin \frac{(N-1) \pi}{N} \mathbf{p}_{N}\right] .
$$

Therefore by (A.26, A.27, A.28) we obtain

$$
\mathbf{Q}^{T} \mathbf{E P}=-\frac{\operatorname{csch} \beta}{\sqrt{D}}\left[\begin{array}{ccccc}
0 & 0 & 0 & \ldots & 0 \\
0 & a_{2} \sin \frac{\pi}{N} & 0 & \ldots & 0 \\
0 & 0 & a_{3} \sin \frac{2 \pi}{N} & \ldots & 0 \\
\ldots & & 0 & \ldots & a_{N} \sin \frac{(N-1) \pi}{N} \\
0 & 0 & 0 &
\end{array}\right]
$$

If we write $\mathbf{F}=\mathbf{Q}^{T} \mathbf{E P}$ and observe the first columns of the two sides of the equation $\mathbf{E P}=\mathbf{Q F}$, then we find that

$$
\sum_{k=1}^{N} e_{j k}=0, j=1,2, \ldots, N
$$


Thus $f_{j j}$ defined in (A.24) are all 0 and (A.22) simplifies to

$$
\mathbf{B}=\iota \mathbf{E} .
$$

So we find that

$$
\mathbf{P}^{T} \mathbf{B Q}=\left[\begin{array}{cccc}
b_{1} & 0 & \ldots & 0 \\
0 & b_{2} & \ldots & 0 \\
\ldots & & & \\
0 & 0 & \ldots & b_{N}
\end{array}\right]
$$

where

$$
b_{j}=-\frac{\iota \operatorname{csch} \beta \sin \frac{\pi(j-1)}{N}}{2 D\left(\operatorname{coth} \beta-\operatorname{csch} \beta \cos \left(\frac{\pi(j-1)}{N}\right)\right)}, j=1,2, \ldots, N
$$

Hence

$$
\left[\begin{array}{rr}
\mathbf{P} & \mathbf{0} \\
\mathbf{0} & \mathbf{Q}
\end{array}\right]^{T} \mathbf{H}\left[\begin{array}{rr}
\mathbf{P} & \mathbf{0} \\
\mathbf{0} & \mathbf{Q}
\end{array}\right]=\left[\begin{array}{llllllll}
a_{1} & 0 & \ldots & 0 & b_{1} & 0 & \ldots & 0 \\
0 & a_{2} & \ldots & 0 & 0 & b_{2} & \ldots & 0 \\
\ldots & & & & & & & \\
0 & 0 & \ldots & a_{N} & 0 & 0 & \ldots & b_{N} \\
b_{1} & 0 & \ldots & 0 & c_{1} & 0 & \ldots & 0 \\
0 & b_{2} & \ldots & 0 & 0 & c_{2} & \ldots & 0 \\
\ldots & & & & & & & \\
0 & 0 & \ldots & b_{N} & 0 & 0 & \ldots & c_{N}
\end{array}\right]
$$

which is indeed a matrix of diagonal $2 \times 2$ blocks

$$
\left[\begin{array}{ll}
a_{j} & b_{j} \\
b_{j} & c_{j}
\end{array}\right]
$$

after we re-label the rows and the columns in (A.34). For (A.35) we already know that $a_{j}$ and $c_{j}$ are positive (A.8, A.19, A.20). A bit more calculations using (A.8, A.19, A.20, A.33) show that the determinants of the $2 \times 2$ blocks (A.35) are again positive. Hence all the eigenvalues of $\mathbf{H}$ are positive.

\section{References}

[1] F. S. Bates and G. H. Fredrickson. Block copolymers - designer soft materials. Physics Today, 52(2):32-38, 1999.

[2] R. Choksi. Scaling laws in microphase separation of diblock copolymers. J. Nonlinear Sci., 11(3):223-236, 2001.

[3] R. Choksi and X. Ren. On the derivation of a density functional theory for microphase separation of diblock copolymers. J. Statist. Phys., 113(1-2):151-176, 2003.

[4] E. De Giorgi. Sulla convergenza di alcune successioni d'integrali del tipo dell'area. Rend. Mat. (6), 8:277-294, 1975.

[5] P. C. Fife and D. Hilhorst. The Nishiura-Ohnishi free boundary problem in the 1D case. SIAM J. Math. Anal., 33(3):589-606, 2001. 
[6] C. Gui and J. Wei. Multiple interior peak solutions for some singular perturbation problems. J. Diff. Eqns., 158:1-27, 1999.

[7] M. Henry. Singular limit of a fourth order problem arising in the micro-phase separation of diblock copolymers. Adv. Differential Equations, 6(9):1049-1114, 2001.

[8] D. Iron, M. J. Ward, and J. Wei. The stability of spike solutions to the one-dimensional Gierer-Meinhardt model. Phys. D, 150(1-2):25-62, 2001.

[9] R. Kohn and P. Sternberg. Local minimisers and singular perturbations. Proc. Roy. Soc. Edinburgh Sect. A, 111(1-2):69-84, 1989.

[10] L. Modica. The gradient theory of phase transitions and the minimal interface criterion. Arch. Rat. Mech. Anal., 98(2):123-142, 1987.

[11] L. Modica and S. Mortola. Un esempio di $\Gamma^{-}$-convergenza. Boll. Un. Mat. Ital. B (5), 14(1):285$299,1977$.

[12] H. Nakazawa and T. Ohta. Microphase separation of ABC-type triblock copolymers. Macromolecules, 26(20):5503-5511, 1993.

[13] Y. Nishiura and I. Ohnishi. Some mathematical aspects of the microphase separation in diblock copolymers. Physica D, 84:31-39, 1995.

[14] I. Ohnishi, Y. Nishiura, M. Imai, and Y. Matsushita. Analytical solutions describing the phase separation driven by a free energy functional containing a long-range interaction term. Chaos, 9(2):329-341, 1999.

[15] T. Ohta and K. Kawasaki. Equilibrium morphology of block copolymer melts. Macromolecules, 19(10):2621-2632, 1986.

[16] X. Ren and J. Wei. On the multiplicity of solutions of two nonlocal variational problems. SIAM J. Math. Anal., 31(4):909-924, 2000.

[17] X. Ren and J. Wei. Concentrically layered energy equilibria of the di-block copolymer problem. European J. Appl. Math., 13(5):479-496, 2002.

[18] X. Ren and J. Wei. On energy minimizers of the di-block copolymer problem. Interfaces Free Bound., 5(2):193-238, 2003.

[19] X. Ren and J. Wei. On the spectra of 3-D lamellar solutions of the diblock copolymer problem. SIAM J. Math. Anal., 35(1):1-32, 2003.

[20] X. Ren and J. Wei. Triblock copolymer theory: Free energy, disordered phase and weak segregation. Physica D, 178(1-2):103-117, 2003.

[21] X. Ren and J. Wei. Triblock copolymer theory: Ordered ABC lamellar phase. J. Nonlinear Sci., 13(2):175-208, 2003.

[22] X. Ren and J. Wei. Stability of spot and ring solutions of the diblock copolymer equation. $J$. Math. Phys., 45(11):4106-4133, 2004. 
[23] X. Ren and J. Wei. Nucleation in the FitzHugh-Nagumo system: interface-spike solutions. $J$. Diff. Eqns., 209(2):266-301, 2005.

[24] X. Ren and J. Wei. Wriggled lamellar solutions and their stability in the diblock copolymer problem. SIAM J. Math. Anal., in press.

[25] X. Ren and J. Wei. Sphere and spherical lamellar patterns in the Ohta-Kawasaki theory of diblock copolymer melts. preprint.

[26] T. Teramoto and Y. Nishiura. Double gyroid morphology in a gradient system with nonlocal effects. Journal of the Physical Society of Japan, 71(7):1611-1614, 2002.

[27] J. Wei and M. Winter. Existence, classification and stability analysis of multiple-peaked solutions for the Gierer-Meinhardt system in $R^{1}$. preprint. 